\title{
Enantioselective Synthesis of the C18-C25 Segment of Lasonolide A by an Oxonia-Cope Prins Cascade
}

\author{
Jackline E. Dalgard and Scott D. Rychnovsky* \\ Department of Chemistry, 516 Rowland Hall, University of \\ California, Irvine, CA 92697.
}

\section{Experimental Procedures and Compound Characterization}

\section{General Experiment Details}

All moisture-and air-sensitive reactions were carried out in flame- or oven-dried glassware using magnetic stirring under a positive pressure of argon gas. Standard syringe/septa techniques were employed. Reaction solvents were distilled or obtained from an alumina filtration system when necessary. Thin layer chromatography was performed on Whatman silica gel PE SIL G/UV plates. Concentration of organic solutions was performed using a Büchi rotary evaporator. Flash chromatography was performed on EM Science 230-400 mesh silica gel. Infrared spectra were recorded on a MIDAC Grams/Prospect FT-IR. NMR spectra were recorded on Brüker GN 500, Brüker Omega 500, and Brüker DRX 400 MHz FTNMR instruments. Proton NMR spectra were obtained using $\mathrm{CDCl}_{3}$ as solvent and referenced to residual protiated solvent ( $\left.\delta 7.26 \mathrm{ppm}\right)$ or $\mathrm{C}_{6} \mathrm{D}_{6}$ and referenced to $\delta 7.16 \mathrm{ppm}$. Carbon NMR spectra were recorded in ppm relative to the residual solvent signal: $\mathrm{CDCl}_{3}(\delta 77.0 \mathrm{ppm})$ or $\mathrm{C}_{6} \mathrm{D}_{6}(\delta 128.4 \mathrm{ppm})$. Mass spectra were determined on an AE2-MS 30, a PG 7070E-HF, a CG Analytical 7070E, or a Fisions autospec spectrometer. Optical rotations were measured with a Jasco DIP-370 
digital polarimeter. Tetrahydrofuran, ethyl ether and methylene chloride were dried by filtration through alumina according to the procedure by Grubbs. ${ }^{1}$ Capillary GC analysis was performed on a Hewlett Packard Model 6890 instrument equipped with a FID detector. All reagents were purchased from Aldrich Chemical Co. or Acros and were used as received, unless otherwise stated. Elemental analyses were performed by M-HW Laboratories (Phoenix, AZ).

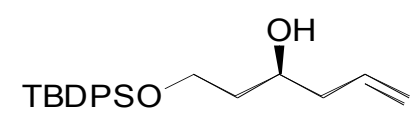

Alcohol 11. ${ }^{2}$ 93\% ee by chiral HPLC; $[\alpha]^{22}{ }_{\mathrm{D}}-3.7\left(c 1.19, \mathrm{CHCl}_{3}\right) ;{ }^{1} \mathrm{H}$ NMR $(500 \mathrm{MHz}$, $\left.\mathrm{CDCl}_{3}\right) \delta$ 7.71-7.68 (m, $\left.4 \mathrm{H}\right), 7.47-7.39(\mathrm{~m}, 6 \mathrm{H}), 5.87$ (dddd, $J=17.1,10.2,7.1,7.1 \mathrm{~Hz}$, $1 \mathrm{H}), 5.16-5.08(\mathrm{~m}, 2 \mathrm{H}), 4.02-3.95(\mathrm{~m}, 1 \mathrm{H}), 3.94-3.83(\mathrm{~m}, 2 \mathrm{H}), 3.0^{\prime}(\mathrm{br} \mathrm{s}, 1 \mathrm{H}), 2.34-$ 2.23 (m, $2 \mathrm{H}), 1.79-1.67$ (m, $2 \mathrm{H}), 1.08(\mathrm{~s}, 9 \mathrm{H}) ;{ }^{13} \mathrm{C} \mathrm{NMR}\left(125 \mathrm{MHz}, \mathrm{CDCl}_{3}\right) \delta$ 135.5, $134.9,133.1,133.0,129.8,129.8,127.8,127.8,127.8,127.8,117.4,70.8,63.2,42.0$, 37.9, 26.8, 19.0. Anal. Calcd for $\mathrm{C}_{22} \mathrm{H}_{30} \mathrm{O}_{2} \mathrm{Si}: \mathrm{C}, 74.53 ; \mathrm{H}, 8.53$. Found: $\mathrm{C}, 74.56 ; \mathrm{H}$, 8.44 .

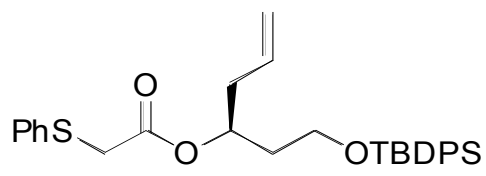

Ester 13. To a stirred solution of alcohol 11 (9.52 g, 26.9 mmol, 1.0 equiv.) in $\mathrm{CH}_{2} \mathrm{Cl}_{2}$ was added $\alpha$-thiophenylacetic acid (4.97 g, $29.5 \mathrm{mmol}, 1.1$ equiv.), DCC (6.1 g, 29.5 mmol, 1.1 equiv.), DMAP·TsOH (749 mg, 2.69 mmol, 0.1 equiv.). The reagents were

\footnotetext{
1 Pangborn, A. B.; Giardello, M. A.; Grubbs, R. H.; Rosen, R. K.; Timmers, F. J. Organometallics 1996, 15, 1518-1520.

2 Alcohol 11: Kabat, M. M.; Wicha, J. Tetrahedron Lett. 1991, 32, 1073-1076.
} 
stirred overnight. Purification without workup $\left(10 \rightarrow 15 \rightarrow 20 \%\right.$ EtOAc/hexanes, $R_{\mathrm{f}}=$ $0.63)$ provided $12.8 \mathrm{~g}(86 \%)$ of ester $\mathbf{1 3}$ as a colorless oil: $[\alpha]^{22}{ }_{\mathrm{D}}+12.5\left(c 1.16, \mathrm{CHCl}_{3}\right)$; IR (neat) 2932, 2118, 1733, 1428, 1268, 1112, 823, $703 \mathrm{~cm}^{-1} ;{ }^{1} \mathrm{H}$ NMR $\left(500 \mathrm{MHz}, \mathrm{C}_{6} \mathrm{D}_{6}\right)$ ठ 7.78-7.74 (m, 4 H), 7.32-7.29 (m, 2 H), 7.25-7.22 (m, 6 H), 6.96-6.93 (m, 2 H), 6.89$6.86(\mathrm{~m}, 1 \mathrm{H}), 5.67-5.59(\mathrm{~m}, 1 \mathrm{H}), 5.31-5.26(\mathrm{~m}, 1 \mathrm{H}), 4.96-4.91(\mathrm{~m}, 2 \mathrm{H}), 3.67-3.64$ (m, $2 \mathrm{H}), 3.25$ (s, $2 \mathrm{H}), 2.18-2.16$ (m, $2 \mathrm{H}), 1.73-1.65$ (m, $2 \mathrm{H}) ;{ }^{13} \mathrm{C}$ NMR (125 MHz, $\left.\mathrm{C}_{6} \mathrm{D}_{6}\right) \delta 169.1,136.4,136.3,134.1,130.4,130.4,130.0,129.5,128.7,128.6,128.5$, 128.2, 127.0, 118.3, 72.0, 60.7, 39.2, 36.9, 36.8, 27.4, 19.8; HRMS (EI) calcd for $\left[\mathrm{C}_{30} \mathrm{H}_{36} \mathrm{O}_{3} \mathrm{SSi}+\mathrm{Na}\right]^{+}$527.2052, found 527.2063. Anal. Calcd for $\mathrm{C}_{30} \mathrm{H}_{36} \mathrm{O}_{3} \mathrm{SSi}$ : C, 71.39; H, 7.19. Found: C, 71.51; H, 7.21.

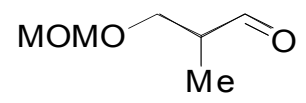

Aldehyde 14. ${ }^{3} \mathrm{H}$ NMR (400 MHz, $\left.\mathrm{CDCl}_{3}\right) \delta 9.73(\mathrm{~d}, J=1.6 \mathrm{~Hz}, 1 \mathrm{H}), 4.61(\mathrm{~s}, 2 \mathrm{H})$, $3.75-3.73(\mathrm{~m}, 2 \mathrm{H}), 3.35(\mathrm{~s}, 3 \mathrm{H}), 2.66-2.62(\mathrm{~m}, 1 \mathrm{H}), 1.15(\mathrm{~d}, J=7.1 \mathrm{~Hz}, 3 \mathrm{H}) ;{ }^{13} \mathrm{C}$ $\operatorname{NMR}\left(100 \mathrm{MHz}, \mathrm{CDCl}_{3}\right) \delta 203.4,96.5,67.4,55.1,46.5,10.5$.

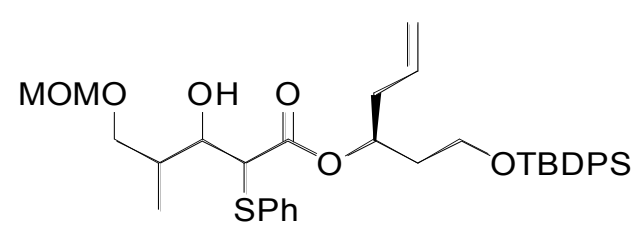

$\beta$-Hydroxy Ester 101. $n$-BuLi (4.0 mL, 2.37 M in hexanes, $9.50 \mathrm{mmol}, 1.1$ equiv.) was added dropwise to a cooled $\left(0{ }^{\circ} \mathrm{C}\right)$ solution of $i \operatorname{Pr}_{2} \mathrm{NH}(1.3 \mathrm{~mL}, 9.50 \mathrm{mmol}, 1.1$ equiv. $)$ in THF (22 mL). After $30 \mathrm{~min}$, the LDA solution was cooled to $-78{ }^{\circ} \mathrm{C}$ and ester 13 (4.36

\footnotetext{
3 Aldehyde 14: Boeckman, R. K.; Enholm, E. J.; Demko, D. M.; Charette, A. B. J. Org. Chem. 1984, $51,4743-4745$.
} 
g, 8.64 mmol, 1.0 equiv.) was added. The mixture was stirred at $-78^{\circ}$ for $1 \mathrm{~h}$, after which time aldehyde 14 (1.37 g, $10.4 \mathrm{mmol}, 1.1$ equiv.) was added. The mixture was stirred an additional $45 \mathrm{~min}$ then warmed to $-35{ }^{\circ} \mathrm{C}$ for $1 \mathrm{~h}$, and the reaction was quenched with satd. aq. $\mathrm{NaHCO}_{3}$. The aqueous layer was extracted with EtOAc, and the organic layers washed with brine, dried $\left(\mathrm{MgSO}_{4}\right)$ and concentrated. Purification by column choromatography provided $2.7 \mathrm{~g}(94 \%)$ of $\mathbf{1 0 1}$ as a mixture of diastereomers: IR (neat) $3502,2931,1729,1428,1111,1045,823,703 \mathrm{~cm}^{-1} ;{ }^{1} \mathrm{H}$ NMR $\left(400 \mathrm{MHz}, \mathrm{CDCl}_{3}\right) \delta$ 7.66-7.61 (m, 4 H), 7.47-7.33 (m, 8 H), 7.28-7.19 (m, 3 H), 5.77-5.60 (m, $1 \mathrm{H}), 5.22-$ 5.14 (m, $1 \mathrm{H}), 5.06-4.99$ (m, $2 \mathrm{H}), 4.62-4.50$ (m, $2 \mathrm{H}), 3.95-3.79$ (m, $2 \mathrm{H}), 3.71-3.52$ (m, 4 H), 3.34-3.29 (m, 3 H), 2.40-2.13 (m, 2 H), 1.84-1.69 (m, 2 H), 1.32-1.24 (m, 1 H), 1.04 (s, $4.5 \mathrm{H}), 1.03(\mathrm{~s}, 4.5 \mathrm{H}), 0.98-0.94(\mathrm{~m}, 1.5 \mathrm{H}), 0.91-0.86$ (m, $1.5 \mathrm{H}) ;{ }^{13} \mathrm{C}$ NMR $\left(100 \mathrm{MHz}, \mathrm{CDCl}_{3}\right) \delta 171.3,171.3,135.5,133.6,133.4,133.3,132.2,132.1,132.1,129.6$, 129.6, 129.0, 129.0, 129.0, 127.7, 127.6, 118.1, 118.0, 117.9, 96.6, 96.5, 96.5, 74.9, 72.1, $72.0,69.3,69.2,60.1,60.0,55.4,55.3,54.6,54.2,38.5,38.3,36.2,36.1,36.0,35.4,35.4$, 31.6, 26.8, 22.6, 19.1, 15.2, 14.7, 14.1; HRMS (EI) calcd for $\left[\mathrm{C}_{36} \mathrm{H}_{48} \mathrm{O}_{6} \mathrm{SSi}+\mathrm{Na}\right]^{+}$ 659.2839 , found 659.2850 .

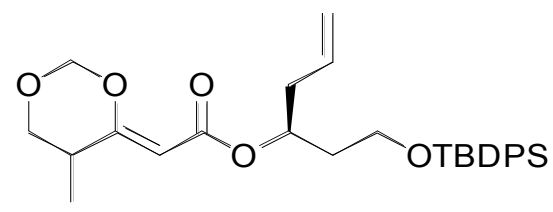

Enol Ether 16. $\mathrm{BF}_{3} \cdot \mathrm{OEt}_{2}(2 \mu \mathrm{L}, 17 \mu \mathrm{mol}, 0.5$ equiv. $)$ was added to a cooled $\left(0{ }^{\circ} \mathrm{C}\right)$ solution of $\beta$-hydroxy ester 101 (16 mg, $35 \mu \mathrm{mol}, 1.0$ equiv.) in $\mathrm{CH}_{2} \mathrm{Cl}_{2}(1 \mathrm{~mL})$. The mixture was stirred at this temperature for $15 \mathrm{~min}$, and then the reaction was quenched with $1 \mathrm{~mL}$ of satd. aq. $\mathrm{NaHCO}_{3}$. The mixture was extracted with diethyl ether $(3 \times 5 \mathrm{~mL})$ 
and washed with brine $(1 \times 5 \mathrm{~mL})$. The organic layers were dried over $\mathrm{MgSO}_{4}$ and concentrated to provide $15 \mathrm{mg}$ of $\mathbf{1 5}$ as a colorless oil.

Sulfide 15 (122 mg, 0.29 mmol, 1.0 equiv.) was dissolved in $\mathrm{CH}_{2} \mathrm{Cl}_{2}(6 \mathrm{~mL})$ and cooled to $-78{ }^{\circ} \mathrm{C} . \mathrm{m}$-CPBA ( $71 \mathrm{mg}, 70 \% \mathrm{wt}, 0.29 \mathrm{mmol}, 1.0$ equiv.) was then added and the reaction mixture was stirred for $25 \mathrm{~min}$. The mixture was diluted with $\mathrm{CH}_{2} \mathrm{Cl}_{2}$, washed with satd. aq. $\mathrm{NaHCO}_{3}, \mathrm{H}_{2} \mathrm{O}$ and brine. The organic layers were dried over $\mathrm{MgSO}_{4}$ and concentrated to provide $116 \mathrm{mg}(91 \%)$ of sulfoxide $\mathbf{1 0 2}$ as a colorless oil. To a solution of sulfoxide $\mathbf{1 0 2}(3 \mathrm{mg}, 48 \mu \mathrm{mol}, 1.0$ equiv.) in $1 \mathrm{~mL}$ of $m$-xylene was added $\mathrm{P}(\mathrm{OEt})_{3}(1 \mu \mathrm{mol}, 48 \mu \mathrm{mol}, 1.0$ equiv. $)$. The reaction mixture was heated at reflux for $1 \mathrm{~h}$ the cooled to room temperature. Purification by column chromatography ( $30 \%$ EtOAc/hexanes, $\mathrm{R}_{\mathrm{f}}=0.66$ in $50 \%$ EtOAc/hexanes) provided $2.2 \mathrm{mg}$ of the $\mathbf{1 6}$ as a mixture of alkene isomers: IR (neat) 2932, 1719, 1646, 1004, 823, $704 \mathrm{~cm}^{-1} ;{ }^{1} \mathrm{H}$ NMR (500 MHz, $\left.\mathrm{C}_{6} \mathrm{D}_{6}\right) \delta$ 7.83-7.78 (m, $\left.4 \mathrm{H}\right), 7.28-7.22(\mathrm{~m}, 6 \mathrm{H}), 5.86$ (dddd, $J=17.1,10.2$, 7.0, $7.0 \mathrm{~Hz}, 1 \mathrm{H}), 5.53-5.43(\mathrm{~m}, 1 \mathrm{H}), 5.06-4.99(\mathrm{~m}, 3 \mathrm{H}), 4.76(\mathrm{~d}, J=5.4 \mathrm{~Hz}, 0.4 \mathrm{H})$, $4.74(\mathrm{~d}, J=5.5 \mathrm{~Hz}, 0.6 \mathrm{H}), 4.67(\mathrm{~d}, J=5.5 \mathrm{~Hz}, 0.6 \mathrm{H}), 4.65(\mathrm{~d}, J=5.5 \mathrm{~Hz}, 0.4 \mathrm{H}), 3.90-$ 3.77 (m, 2 H), 3.36-3.30 (m, 1 H), 2.97-2.91 (m, 1 H), 2.41-2.37 (m, 2 H), 2.02-1.93 (m, $1 \mathrm{H}), 1.92-1.87$ (m, $2 \mathrm{H}), 1.19$ (s, $3.6 \mathrm{H}), 1.19(\mathrm{~s}, 5.4 \mathrm{H}), 0.57$ (d, $J=6.8 \mathrm{~Hz}, 1.8 \mathrm{H})$, $0.54(\mathrm{~d}, J=6.8 \mathrm{~Hz}, 1.2 \mathrm{H}) ;{ }^{13} \mathrm{C} \mathrm{NMR}\left(125 \mathrm{MHz}, \mathrm{C}_{6} \mathrm{D}_{6}\right) \delta 168.4,164.3,136.5,136.4$, $134.8,134.6,130.3,130.2,128.7,128.6,128.5,128.2,117.9,98.9,93.4,93.4,71.0,69.9$, $69.9, \quad 61.1, \quad 39.6, \quad 37.3, \quad 33.8, \quad 27.5, \quad 19.8, \quad 15.1, \quad 14.9$; HRMS (EI) calcd for $\left[\mathrm{C}_{29} \mathrm{H}_{38} \mathrm{O}_{5} \mathrm{Si}+\mathrm{Na}\right]^{+}$517.2386, found 517.2366. 


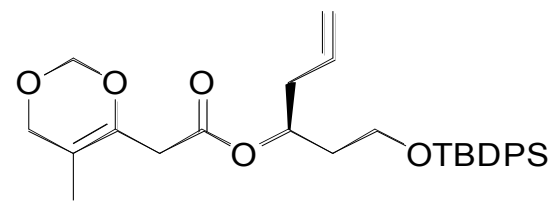

Enol Ether 17. NaHMDS ( $59 \mu \mathrm{L}, 1.0 \mathrm{M}$ in hexanes, $59 \mu \mathrm{mol}, 1.0$ equiv.) was added to a cooled $\left(-78{ }^{\circ} \mathrm{C}\right)$ solution of $\alpha, \beta$-unsaturated ester $16(29 \mathrm{mg}, 59 \mu \mathrm{mol}, 1.0$ equiv. $)$ in 0.5 mL THF. After $1 \mathrm{~min}$, satd. aq. $\mathrm{NaHCO}_{3}(0.5 \mathrm{~mL})$ was added. After extractive workup with ether, the organic layers were washed with brine, dried $\left(\mathrm{MgSO}_{4}\right)$ and concentrated to provide $22 \mathrm{mg}(76 \%)$ of $\mathbf{1 7}$ as a colorless oil: $[\alpha]^{22}{ }_{\mathrm{D}}+12.6\left(c 0.89, \mathrm{CHCl}_{3}\right)$; IR (neat) 2931, 1738, 1428, 1015, 823, $704 \mathrm{~cm}^{-1} ;{ }^{1} \mathrm{H}$ NMR $\left(500 \mathrm{MHz}, \mathrm{C}_{6} \mathrm{D}_{6}\right) \delta$ 7.79-7.75 (m, $\left.4 \mathrm{H}\right)$, 7.28-7.22 (m, $6 \mathrm{H}), 5.71$ (dddd, $J=17.8,9.6,7.1,7.1 \mathrm{~Hz}, 1 \mathrm{H}), 5.37-5.31(\mathrm{~m}, 1 \mathrm{H})$, 5.00-4.95 (m, 2 H), 4.80 (s, 2 H), 3.78-3.76 (m, 2 H), 3.76-3.67 (m, 2 H), 2.99 (s, 2 H), 2.29-2.18 (m, $2 \mathrm{H}), 1.79-1.74$ (m, $2 \mathrm{H}), 1.17$ (s, $9 \mathrm{H}), 1.56$ (s, $3 \mathrm{H}) ;{ }^{13} \mathrm{C}$ NMR (125 MHz, $\left.\mathrm{C}_{6} \mathrm{D}_{6}\right) \delta 169.2,143.1,136.4,136.3,134.5,134.4,134.3,130.4,130.4,128.7,128.6$, $128.5,128.2,118.1,107.4,90.9,71.3,67.5,60.8,39.4,37.1,36.9,27.4,19.8,13.0$; HRMS (EI) calcd for $\left[\mathrm{C}_{29} \mathrm{H}_{38} \mathrm{O}_{5} \mathrm{Si}+\mathrm{Na}\right]^{+}$517.2386, found 517.2391.

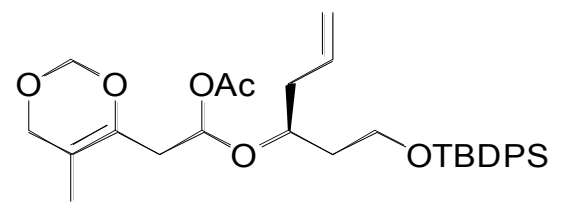

a-Acetoxy Ether 5. DIBAL-H ( $0.87 \mathrm{~mL}, 1.0 \mathrm{M}$ in hexanes, $0.87 \mathrm{mmol}, 1.5$ equiv.) was added to a cooled $\left(-78^{\circ} \mathrm{C}\right)$ solution of ester $17(286 \mathrm{mg}, 0.58 \mathrm{mmol}, 1.0$ equiv.) in $3 \mathrm{~mL}$ of $\mathrm{CH}_{2} \mathrm{Cl}_{2}$. After $50 \mathrm{~min}$, pyridine ( $0.12 \mathrm{~mL}, 1.45 \mathrm{mmol}, 2.5$ equiv.), DMAP (177 $\mathrm{mg}$ in $2 \mathrm{~mL} \mathrm{CH}_{2} \mathrm{Cl}_{2}, 1.45 \mathrm{mmol}, 2.5$ equiv.) and acetic anhydride $(0.32 \mathrm{~mL}, 2.89 \mathrm{mmol}, 5.0$ equiv.) were added sequentially. The reaction mixture was stirred at $-78{ }^{\circ} \mathrm{C}$ for $16 \mathrm{~h}$ then 
warmed to $-10{ }^{\circ} \mathrm{C}$ over $0.5 \mathrm{~h}$. The reagents were quenched with Rochelle's salt (6 mL) and $\mathrm{H}_{2} \mathrm{O}(6 \mathrm{~mL})$. The mixture was extracted with ether $(3 \times 30 \mathrm{~mL})$, washed with $\mathrm{H}_{2} \mathrm{O}$ and brine. After the organic layers were dried over $\mathrm{MgSO}_{4}$ and concentrated, $285 \mathrm{mg}$ (quantitative yield) of $\mathbf{5}$ was produced as a 1:1 mixture of diastereomers: $[\alpha]^{22}+12.6(c$ 0.68, $\mathrm{CHCl}_{3}$ ); IR (neat) 2933,1741, 1239, 1111, 1010, 823, $704 \mathrm{~cm}^{-1} ;{ }^{1} \mathrm{H}$ NMR (500 $\left.\mathrm{MHz}, \mathrm{C}_{6} \mathrm{D}_{6}\right) \delta$ 7.83-7.75 (m, $\left.4 \mathrm{H}\right), 7.30-7.21(\mathrm{~m}, 6 \mathrm{H}), 6.45-6.40(\mathrm{~m}, 1 \mathrm{H}), 5.96-5.87$ (m, 0.4 H), 5.82-5.73 (m, 0.6 H), 5.05-4.98 (m, 2 H), 4.12-4.06 (m, 0.4 H), 5.82-5.73 (m, 0.6 H), 5.05-4.98 (m, 2 H), 4.85-4.76 (m, 2 H), 4.12-4.06 (m, 0.4 H), 4.03-3.97 (m, 0.6 H), 3.92-3.73 (m, 4 H), 2.64-2.53 (m, 2 H), 2.33-2.18 (m, 2 H), 1.86-1.72 (m, 2 H), 1.71 (s, $1.2 \mathrm{H}), 1.69$ (s, $1.8 \mathrm{H}), 1.24$ (s, $3 \mathrm{H}), 1.19$ (s, $3.6 \mathrm{H}), 1.16$ (s, $5.4 \mathrm{H}) ;{ }^{13} \mathrm{C}$ NMR (125 MHz, $\left.\mathrm{C}_{6} \mathrm{D}_{6}\right) \delta 170.4,170.2,144.3,136.4,136.4,136.3,135.5,134.8,134.7,134.6$, 134.6, 130.4, 130.3, 130.3, 128.7, 128.6, 128.5, 118.1, 117.3, 107.2, 96.9, 95.4, 90.8, $77.1,75.3,67.6,67.5,61.4,60.9,40.4,39.1,38.2,37.8,36.8,36.7,27.5,21.3,21.3,19.8$ HRMS (EI) calcd for $\left[\mathrm{C}_{31} \mathrm{H}_{42} \mathrm{O}_{6} \mathrm{Si}+\mathrm{Na}\right]^{+}$561.2648, found 561.2640.

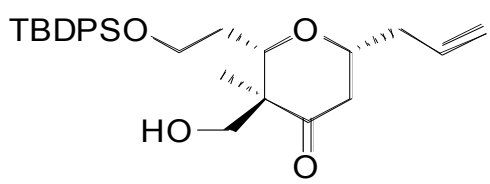

Tetrahydropyranone 10 . TMSOTf $(67 \mu \mathrm{L}, 0.37 \mathrm{mmol}, 3.0$ equiv. $)$ was added to a cooled $\left(-78^{\circ} \mathrm{C}\right)$ solution of $\alpha$-acetoxy ether 5 (66 $\mathrm{mg}, 0.12 \mathrm{mmol}, 1.0$ equiv.) and 2,6-ditert-butyl-4-methylpyridine ( $25 \mathrm{mg}, 0.12 \mathrm{mmol}, 1.0$ equiv.) in $2.5 \mathrm{~mL}$ of $\mathrm{CH}_{2} \mathrm{Cl}_{2}$. After 2 $\mathrm{h}$, the reagents were quenched with $3 \mathrm{~mL}$ satd. aq. $\mathrm{NaHCO}_{3}$, and the mixture was stirred for 10 min. Purification by column chromatography (20\% EtOAc/hexanes) provided 42 $\mathrm{mg}(74 \%)$ of $\mathbf{1 0}$ as a single diastereomer. The enantiomeric excess of $\mathbf{1 0}$ was determined 
to be $93 \%$ by HPLC analysis (Diacel OD-H column with 98:2 Hexanes/IPA solvent mixture and a $0.9 \mathrm{~mL} / \mathrm{min}$ flow rate; $\mathrm{R}_{\mathrm{t}}($ minor $)=9.07$ min and $\mathrm{R}_{\mathrm{t}}($ major $)=10.9 \mathrm{~min}$.) Ketone 10 was isolated as a colorless oil: $[\alpha]_{\mathrm{D}}^{22}-51.4\left(c 0.18, \mathrm{CHCl}_{3}\right)$; IR (neat) 3475, 2929, 1704, 1110, $704 \mathrm{~cm}^{-1} ;{ }^{1} \mathrm{H}$ NMR $\left(500 \mathrm{MHz}, \mathrm{CDCl}_{3}\right) \delta$ 7.68-7.64 (m, $\left.4 \mathrm{H}\right), 7.44-$ 7.35 (m, $6 \mathrm{H}), 5.74$ (dddd, $J=17.2,10.2,7.0,7.0 \mathrm{~Hz}, 1 \mathrm{H}), 5.10-5.04$ (m, $2 \mathrm{H}), 3.89$ $3.80(\mathrm{~m}, 3 \mathrm{H}), 3.74(\mathrm{~d}, J=11.9 \mathrm{~Hz}, 1 \mathrm{H}), 3.59-3.53(\mathrm{~m}, 1 \mathrm{H}), 3.48(\mathrm{~d}, J=11.8 \mathrm{~Hz}, 1 \mathrm{H})$, $2.48(\mathrm{dd}, J=14.4,11.7 \mathrm{~Hz}, 1 \mathrm{H}), 2.35-2.21(\mathrm{~m}, 3 \mathrm{H}), 1.76-1.65$ (m, $2 \mathrm{H}), 1.06$ (s, $3 \mathrm{H})$, 1.04 (s, $9 \mathrm{H}) ;{ }^{13} \mathrm{C}$ NMR $\left(125 \mathrm{MHz}, \mathrm{CDCl}_{3}\right) \delta$ 214.3, 135.6, 133.9, 133.3, 129.6, 127.6, 117.9, 76.3, 76.1, 64.5, 60.6, 53.8, 44.6, 40.4, 32.1, 26.9, 19.2, 14.8; HRMS (EI) calcd for $\left[\mathrm{C}_{28} \mathrm{H}_{38} \mathrm{O}_{4} \mathrm{Si}+\mathrm{Na}\right]^{+}$489.2437, found 489.2417.

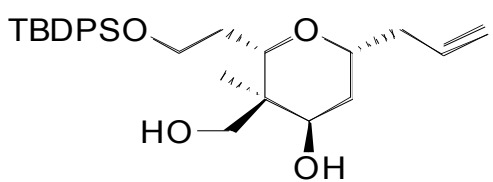

Tetrahydropyran 18. L-Selectride ( $51 \mu \mathrm{L}, 1.0 \mathrm{M}$ in THF, $51 \mu$ mol, 3.0 equiv.) was added to a cooled $\left(-78{ }^{\circ} \mathrm{C}\right)$ solution of tetrahydropyranone $10(9 \mathrm{mg}, 17 \mu \mathrm{mol}, 1.0$ equiv.) in $0.2 \mathrm{~mL}$ of THF. After $45 \mathrm{~min}, 0.5 \mathrm{~mL}$ of $\mathrm{MeOH}, 1 \mathrm{~N} \mathrm{NaOH}$ and $30 \% \mathrm{H}_{2} \mathrm{O}_{2}$ were added sequentially. After warming to RT over $1.5 \mathrm{~h}$, the mixture was diluted with $3 \mathrm{~mL}$ $\mathrm{H}_{2} \mathrm{O}$. The aqueous layer was extracted with EtOAc, and the combined extracts were washed with brine, dried over $\mathrm{MgSO}_{4}$ and concentrated to give $8.4 \mathrm{mg}(94 \%)$ of $\mathbf{1 8}$ as a single diastereomer: $[\alpha]_{D}^{22}-25.3\left(c 0.38, \mathrm{CHCl}_{3}\right.$ ); IR (neat) 3412, 2930, 1643, 1111, 1029, $702 \mathrm{~cm}^{-1} ;{ }^{1} \mathrm{H}$ NMR (500 MHz, $\left.\mathrm{CHCl}_{3}\right) \delta$ 7.70-7.66 (m, $\left.4 \mathrm{H}\right), 7.44-7.36(\mathrm{~m}, 6 \mathrm{H})$, 5.78 (dddd, $J=17.2,10.1,7.1,7.1 \mathrm{~Hz}, 1 \mathrm{H}), 5.07-4.96$ (m, $2 \mathrm{H}), 4.19$ (d, $J=8.4 \mathrm{~Hz}, 1$ H), 3.91-3.90 (m, $1 \mathrm{H}), 3.89-3.81(\mathrm{~m}, 3 \mathrm{H}), 3.69$ (d, $J=11.6 \mathrm{~Hz}, 1 \mathrm{H}), 3.58$ (d, $J=11.6$ 
Hz, $1 \mathrm{H}), 2.46$ (br s, $2 \mathrm{H}), 2.28-2.19$ (m, $1 \mathrm{H}), 2.15-2.08$ (m, $1 \mathrm{H}), 1.77-1.70(\mathrm{~m}, 1 \mathrm{H})$, 1.67-1.60 (m, $1 \mathrm{H}), 1.57-1.51$ (m, $2 \mathrm{H}), 1.06$ (s, $9 \mathrm{H}), 0.77$ (s, $3 \mathrm{H}) ;{ }^{13} \mathrm{C}$ NMR (125 MHz, $\left.\mathrm{CHCl}_{3}\right) \delta 135.6,135.5,134.9,133.8,133.7,129.6,127.6,116.5,74.9,71.5,71.2,69.9$, $61.5,40.2,40.0,35.8,32.4,26.9,19.2,15.3$; HRMS (EI) calcd for $\left[\mathrm{C}_{28} \mathrm{H}_{40} \mathrm{O}_{4} \mathrm{Si}+\mathrm{Na}\right]^{+}$ 491.2594, found 491.2581 .

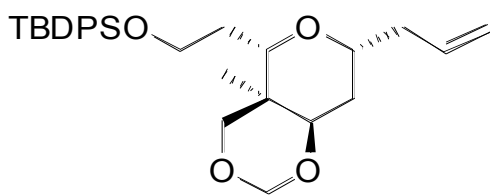

Tetrahydropyran 19. To a cooled $\left(-78{ }^{\circ} \mathrm{C}\right)$ solution of $\alpha$-acetoxy ether $5(12 \mathrm{mg}, 22$

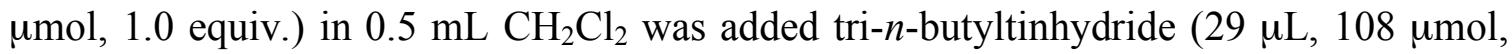
5.0 equiv) followed by TMSOTf ( $12 \mu \mathrm{L}, 65 \mu \mathrm{mol}, 3.0$ equiv.). After $1 \mathrm{~h}$, the reagents were quenched with satd. aq. $\mathrm{NaHCO}_{3}$, and the mixture extracted with diethyl ether. The organics were washed with $\mathrm{H}_{2} \mathrm{O}$ and brine, then dried and concentrated. Purification by column chromatography $\left(20 \%\right.$ diethyl ether/hexanes, $\left.\mathrm{R}_{\mathrm{f}}=0.52\right)$ provided $8 \mathrm{mg}(80 \%)$ of 19 as a 5:1 eq/ax mixture of $\mathrm{C} 4$ epimers: ${ }^{1} \mathrm{H}$ NMR $\left(500 \mathrm{MHz}, \mathrm{C}_{6} \mathrm{D}_{6}\right) \delta$ 7.85-7.83 (m, 4 H), 7.28-7.24 (m, 6 H), 5.86-5.76 (m, $1 \mathrm{H}), 5.09-4.98$ (m, $3 \mathrm{H}), 4.60$ (d, $J=10.4 \mathrm{~Hz}, 1$ H), $4.39(\mathrm{~d}, J=5.8 \mathrm{~Hz}, 1 \mathrm{H}), 4.10-4.04(\mathrm{~m}, 1 \mathrm{H}), 4.00-3.94(\mathrm{~m}, 1 \mathrm{H}), 3.88-3.86(\mathrm{~m}, 1$ H), $3.66(\mathrm{~d}, J=12.1 \mathrm{~Hz}, 1 \mathrm{H}), 3.19(\mathrm{t}, J=2.9 \mathrm{~Hz}, 1 \mathrm{H}), 2.85(\mathrm{~d}, J=11.9 \mathrm{~Hz}, 1 \mathrm{H}), 2.23-$ $2.01(\mathrm{~m}, 2 \mathrm{H}), 1.79-1.71(\mathrm{~m}, 1 \mathrm{H}), 1.53-1.44(\mathrm{~m}, 3 \mathrm{H}), 1.24$ (s, $9 \mathrm{H}), 1.95(\mathrm{~s}, 3 \mathrm{H}) ;{ }^{13} \mathrm{C}$ NMR (125 MHz, $\left.\mathrm{C}_{6} \mathrm{D}_{6}\right) \delta 136.5,135.6,135.2,130.2,128.6,128.6,128.1,116.9,94.6$, 80.2, 73.2, 72.2, 72.1, 61.9, 41.1, 33.1, 27.6; HRMS (EI) calcd for $\left[\mathrm{C}_{29} \mathrm{H}_{40} \mathrm{O}_{4} \mathrm{Si}+\mathrm{H}\right]^{+}$ 481.2774, found 481.2768 . 


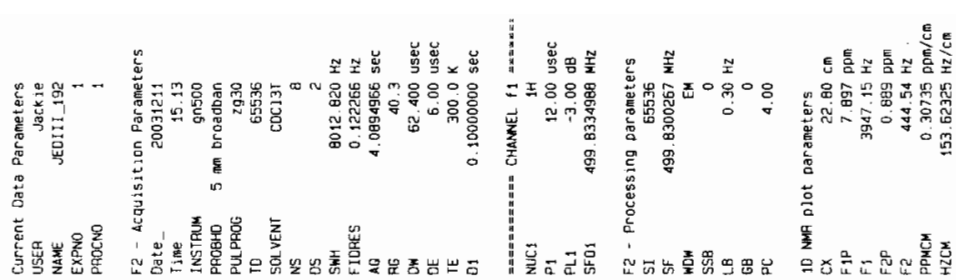

ED2.06 0 -

Etr $\angle 0^{\circ}:$

$\angle 6980$ ' -

8EROL

$\checkmark 5 G L L^{\prime}-$

EEGe L I

Bเze

$0065<$

p585 L : -

\section{$\angle \varepsilon \theta 92 \cdot c$}

टь6/2 ट-

I

89462 ?
E9G0e है
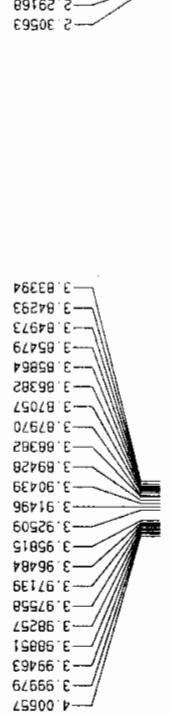

$5<650$ ह
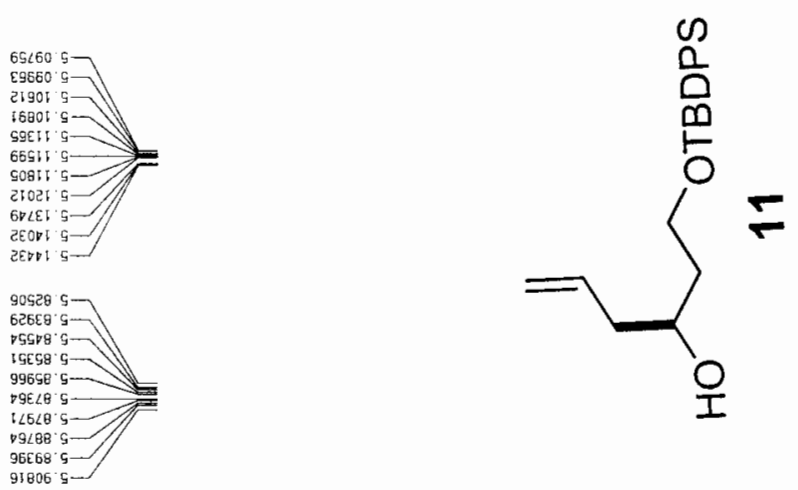

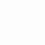

+

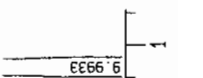



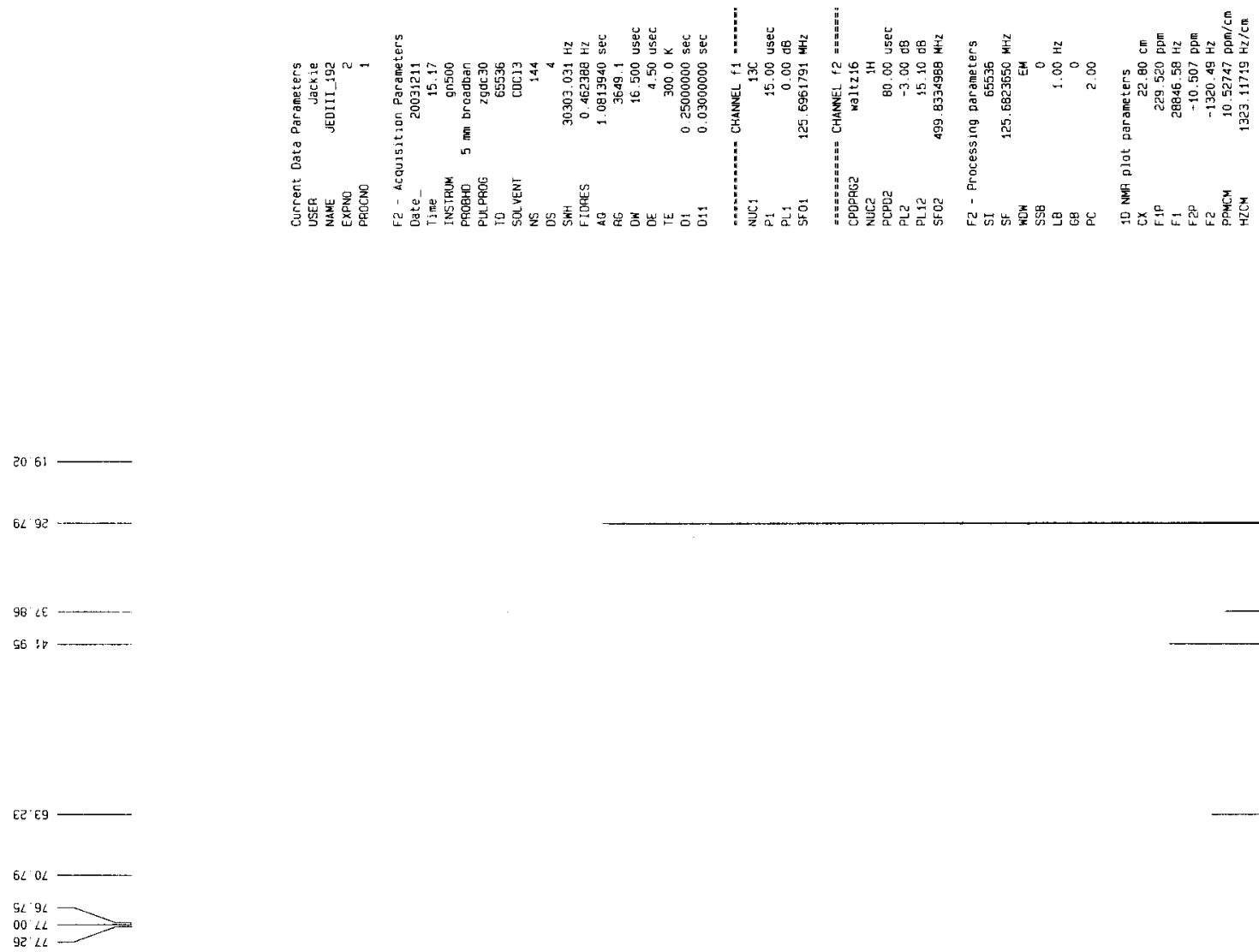

924
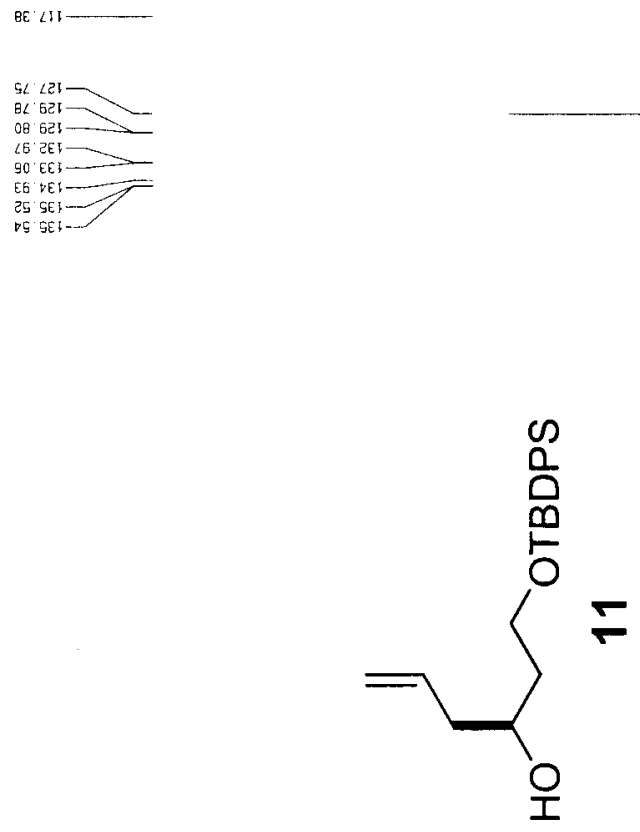


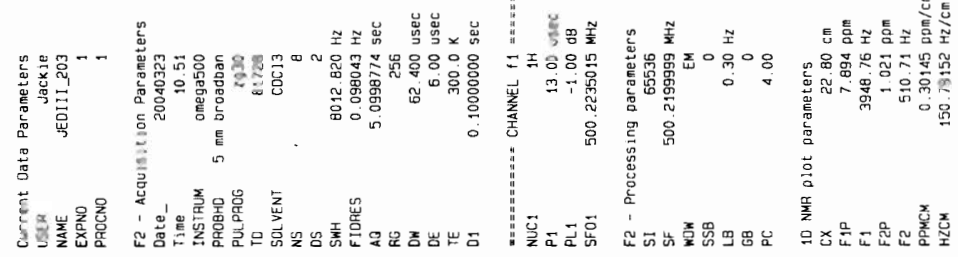

$50991 \cdot$

$\nabla 65 \angle \mathrm{b}$

ocess : -

$56 r 291-$

rco6s: :-

50006

cosk:-

cosst z-

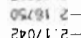

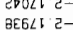

BE 62 .

$\operatorname{scose} \varepsilon-1-$

acres $c$ -

$\sin 8$

trems c-

taxs a-

corso \&-

zis9s ह-

0๖टा6

8І5॰6

a)

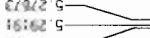

iczoc: 5

560095

Eटt19 5

89819.5
$8 \pi E 29.5$

BLEEg $\mathrm{s}$

BELES 5

EDSG9 5

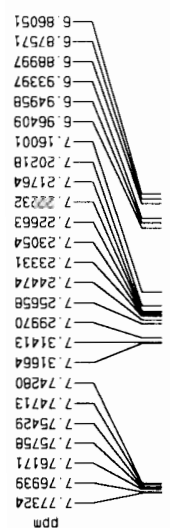

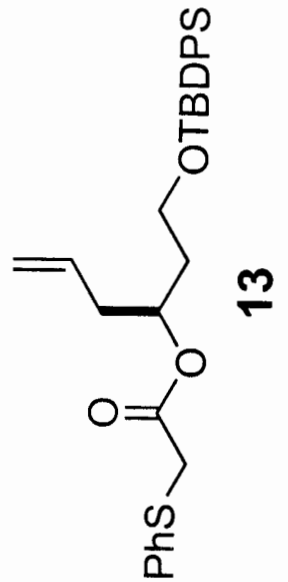

$\bar{\Lambda}$ 

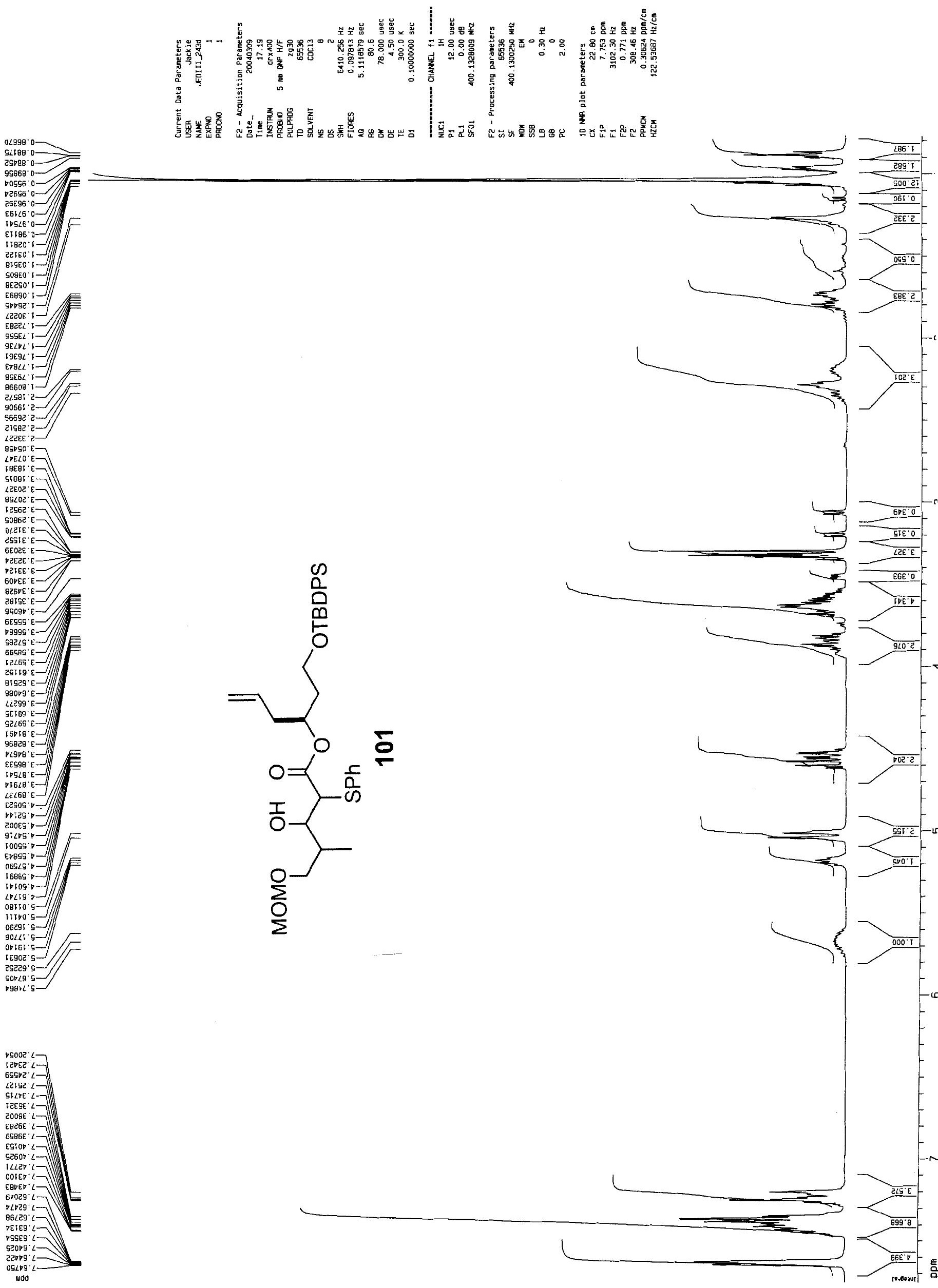


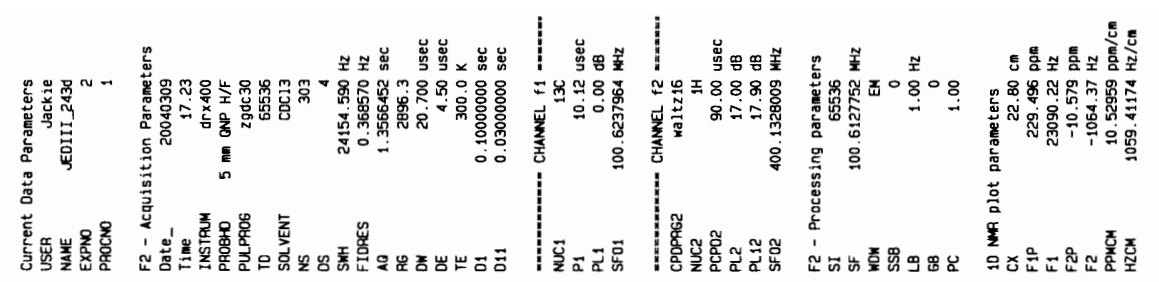

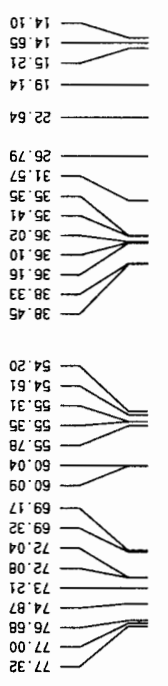

$66 \cdot 96$

$8 G^{\prime} 96$

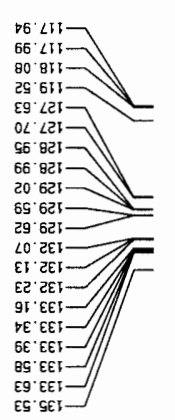

GE' $T \angle L=$

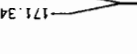

$\angle E^{\prime} 6 \angle I$

$28 \cdot 665$

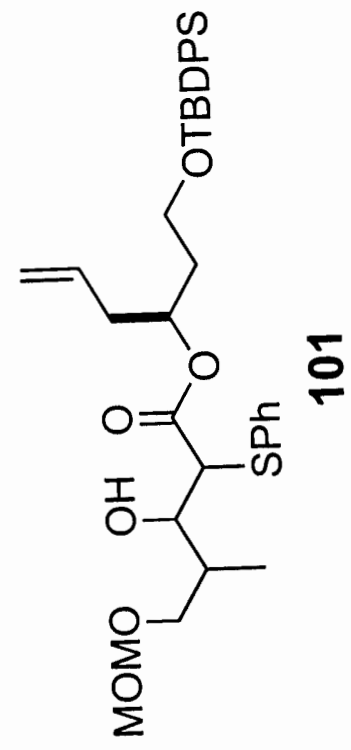

Do. $802-$
in. $012-$

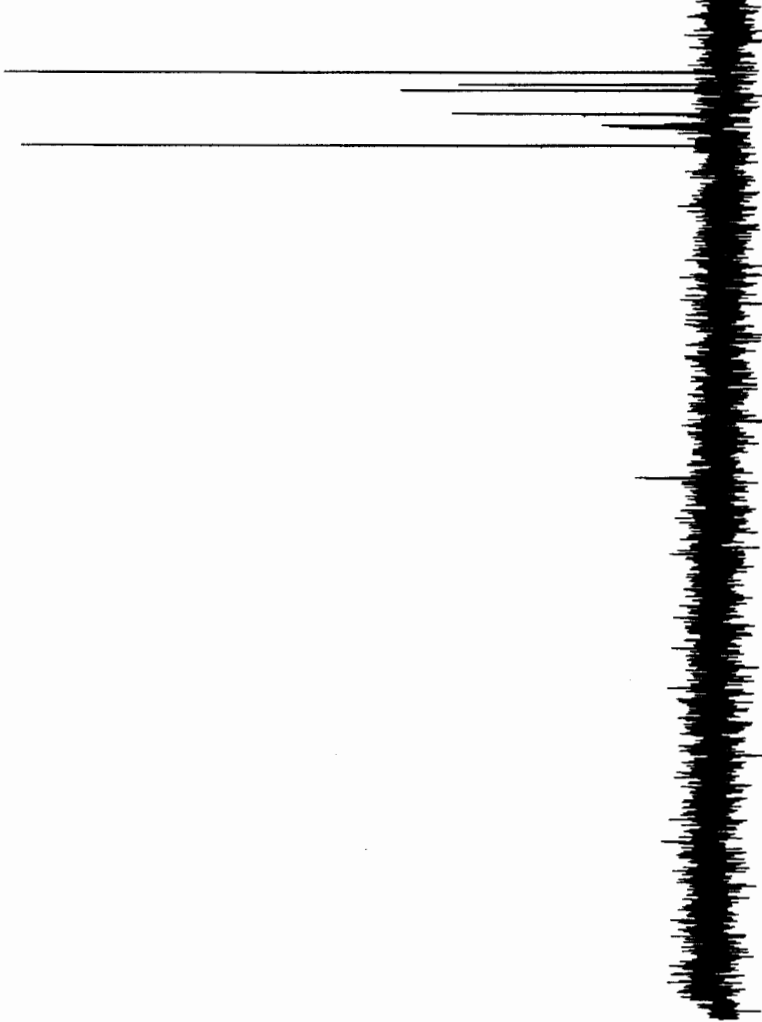



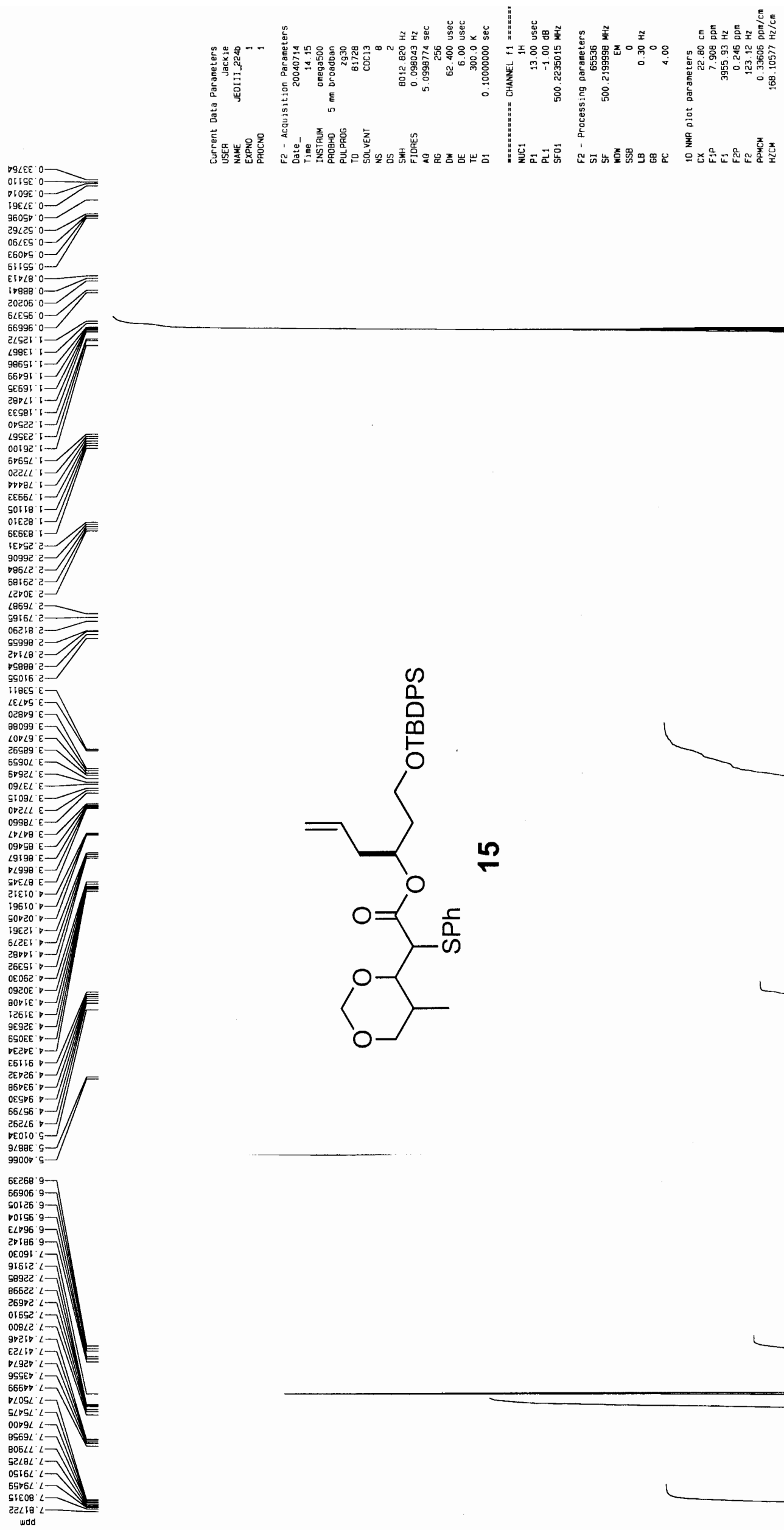

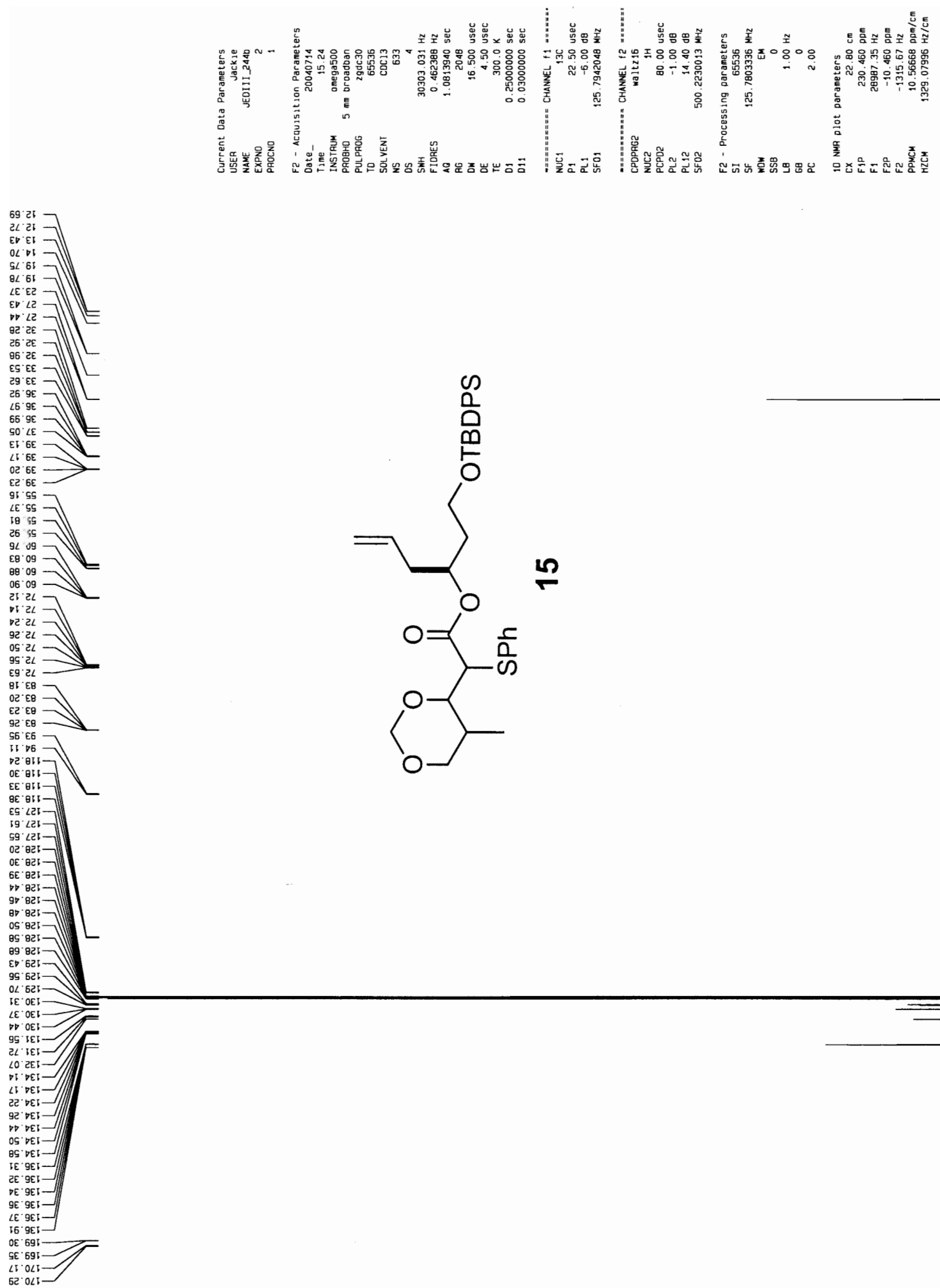

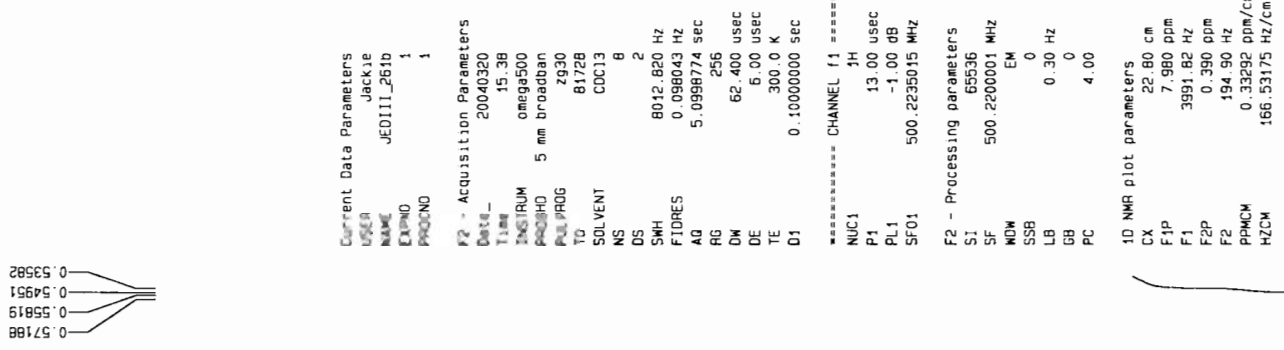

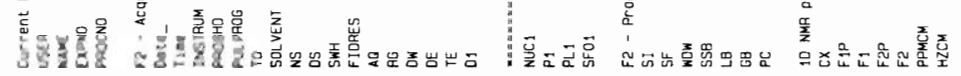

$\varepsilon 688 \%$ :

$02+61 \div \div$

$8 \operatorname{cosg} \cdot \mathrm{t}$

$5 T \forall \angle B^{\circ} \cdot$

$\angle 68 \angle \theta^{\prime} \cdot 1$

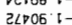

58 เ 16 .

DiLTE'

OSLSE ?

$\angle \angle 0 \angle 6^{\circ}$.

$6 \angle 286^{\circ} \cdot$

905 $\angle E \cdot 2 \longrightarrow$

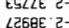

SOLOb 2 -

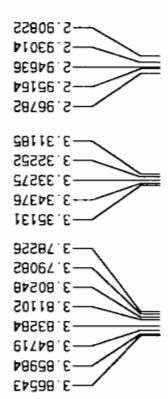

$\angle 9129 \cdot 6-$

LOEटE9 $\cdot$

$\varepsilon 62 \angle 9 \forall-$

$\triangle \triangle 8 L L ' b-$

SLSSL $\square=11$

$09992 b-$

BL266 '

D2L6E' '

$60160^{\circ} \mathrm{s}$

DLEIOSD

$6 \angle 210$.

DDLEO 9

ट8160's

(1)

口ह550.

cesar

EEL60 5

SEIIS 5

90615

$90762 \mathrm{c}$

88t18 5

ज9228 5

29:208.5-

E06P8: 5

ट8958 5-

$66651<-7$

$\begin{array}{lll}66651 & <-7 \\ 80022 & <-7\end{array}$

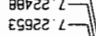

०टिटट $L 7$

DSDEC 2

LSEDe $L$

$16902-$

29992 2

o08Le $\angle$

$00 \varepsilon \theta 2<$

:898L $\angle$

$9568<2<7$

$90952<-$

$08862<$

66108 $\angle$

(2)

(2)

(2)

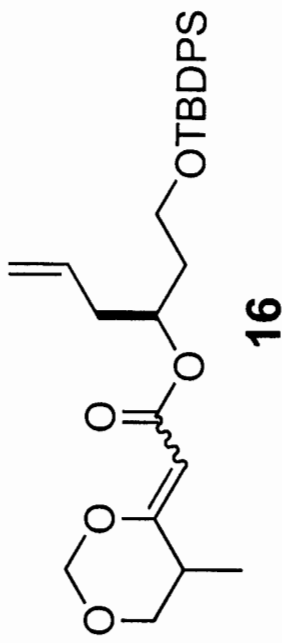

$\mathscr{\sigma}$
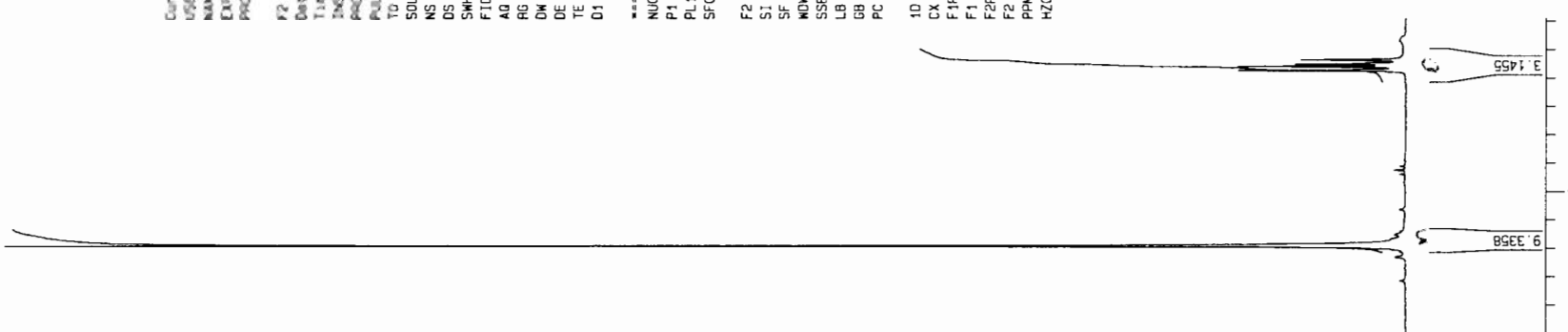

.

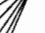




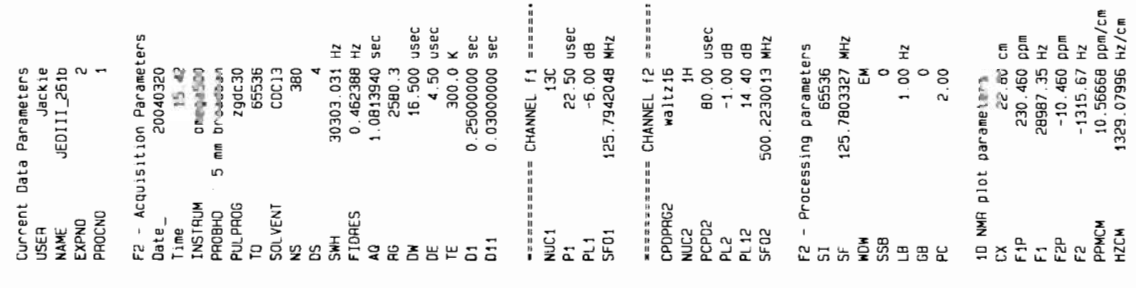

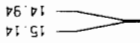

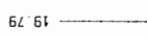

$\angle P L 2$

$\longleftrightarrow \cdot \varepsilon \varepsilon-$
$6 \mathcal{L}\llcorner\varepsilon-\square$
$\square 9 \cdot 6 \varepsilon-\square$

II. 19

58.69

$88.69 \longrightarrow$

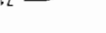

$\left.{ }_{\varepsilon \cdot}^{8 E} \varepsilon 6\right] \longrightarrow$

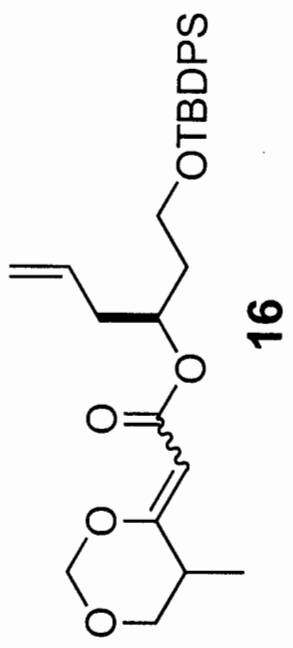

$76^{\circ} 86$ 


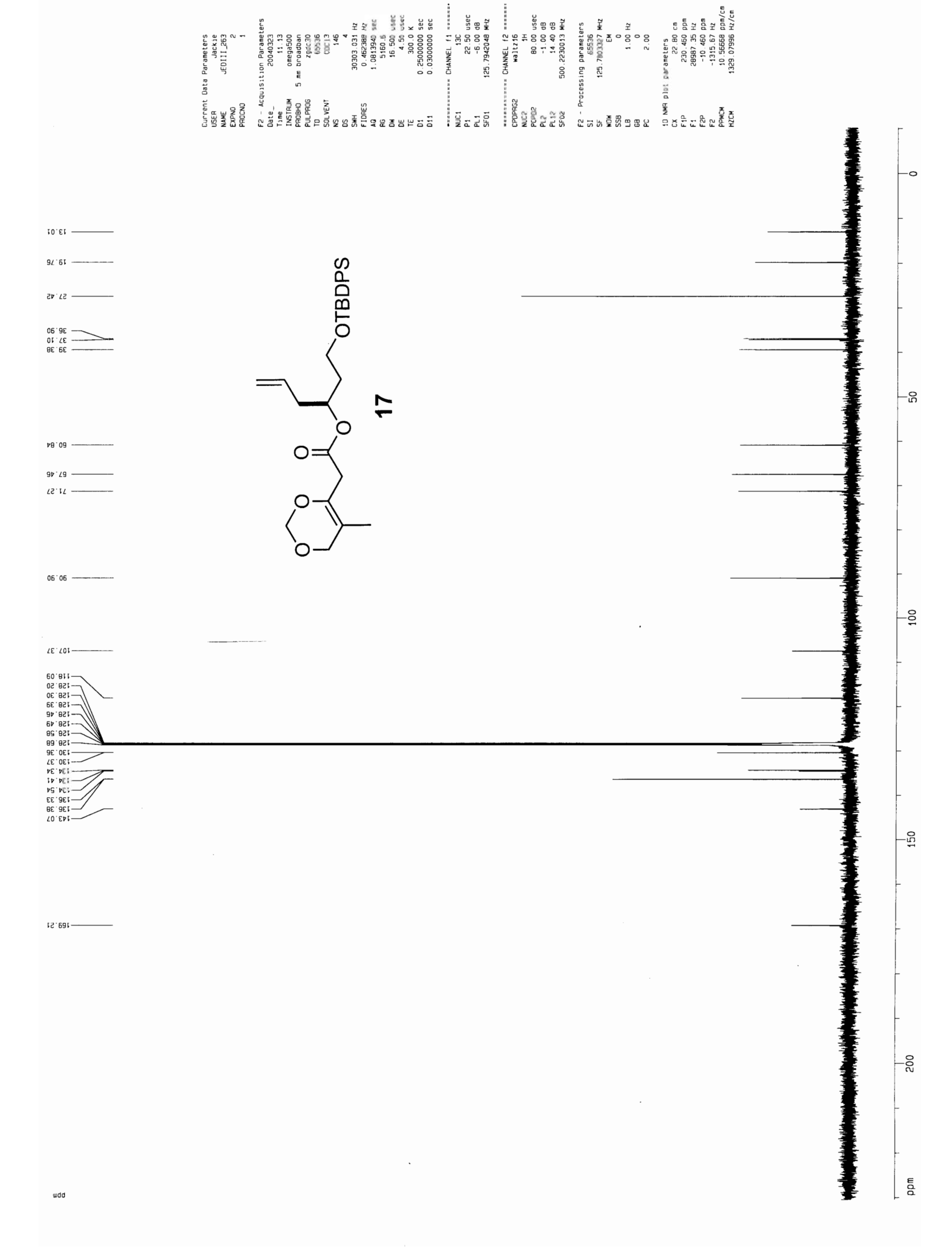



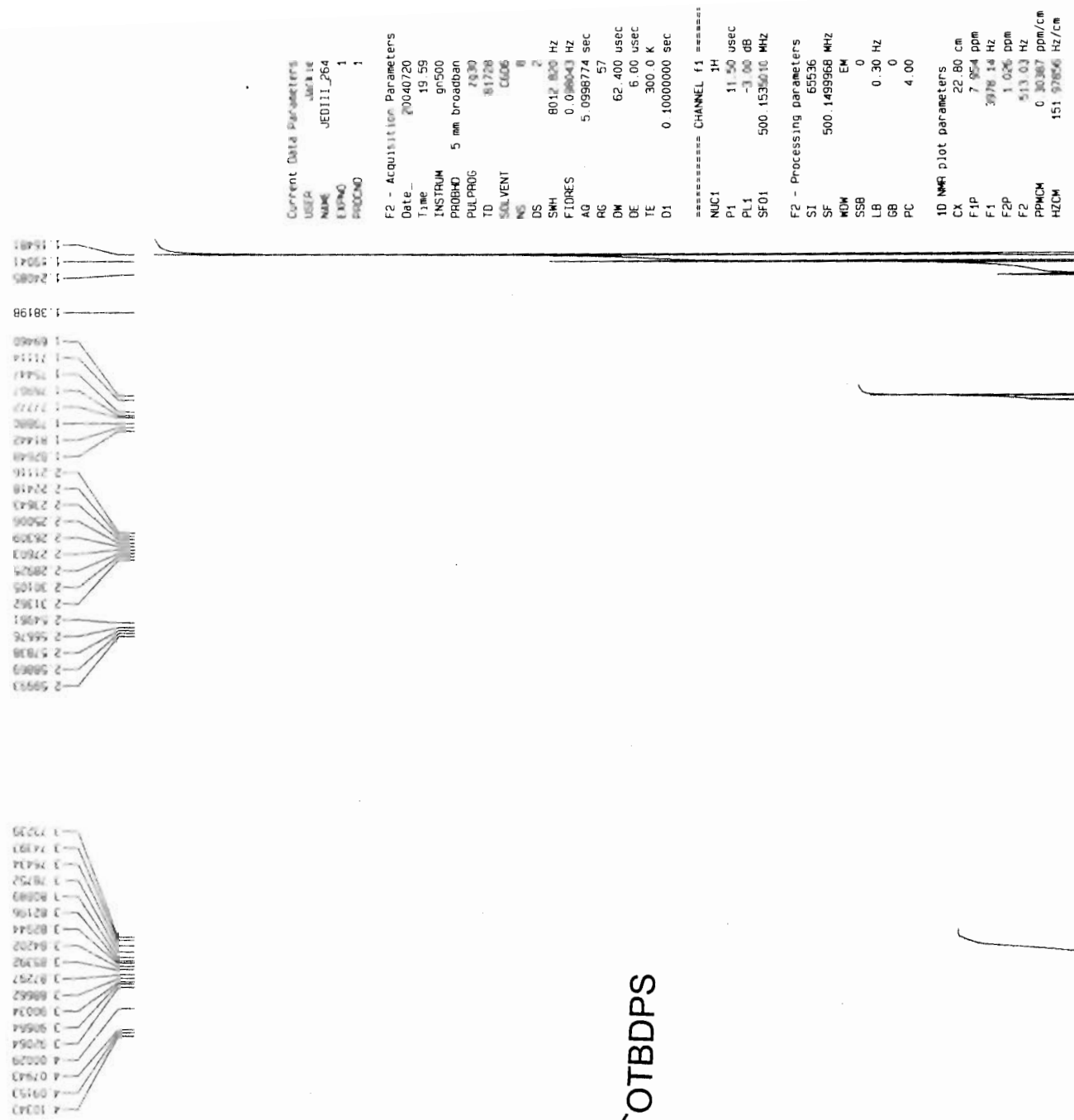

tew: $x$

cores ?

wow $x$

cosien $\times-\ldots$

wow $7-7$

iscrein p-

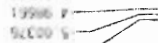

siace?

ocone
sowi
21906

and 5

wex 5 - 5

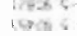

Eitits 9

ascos 4,1,

wary 9

cetcrs $3-1$
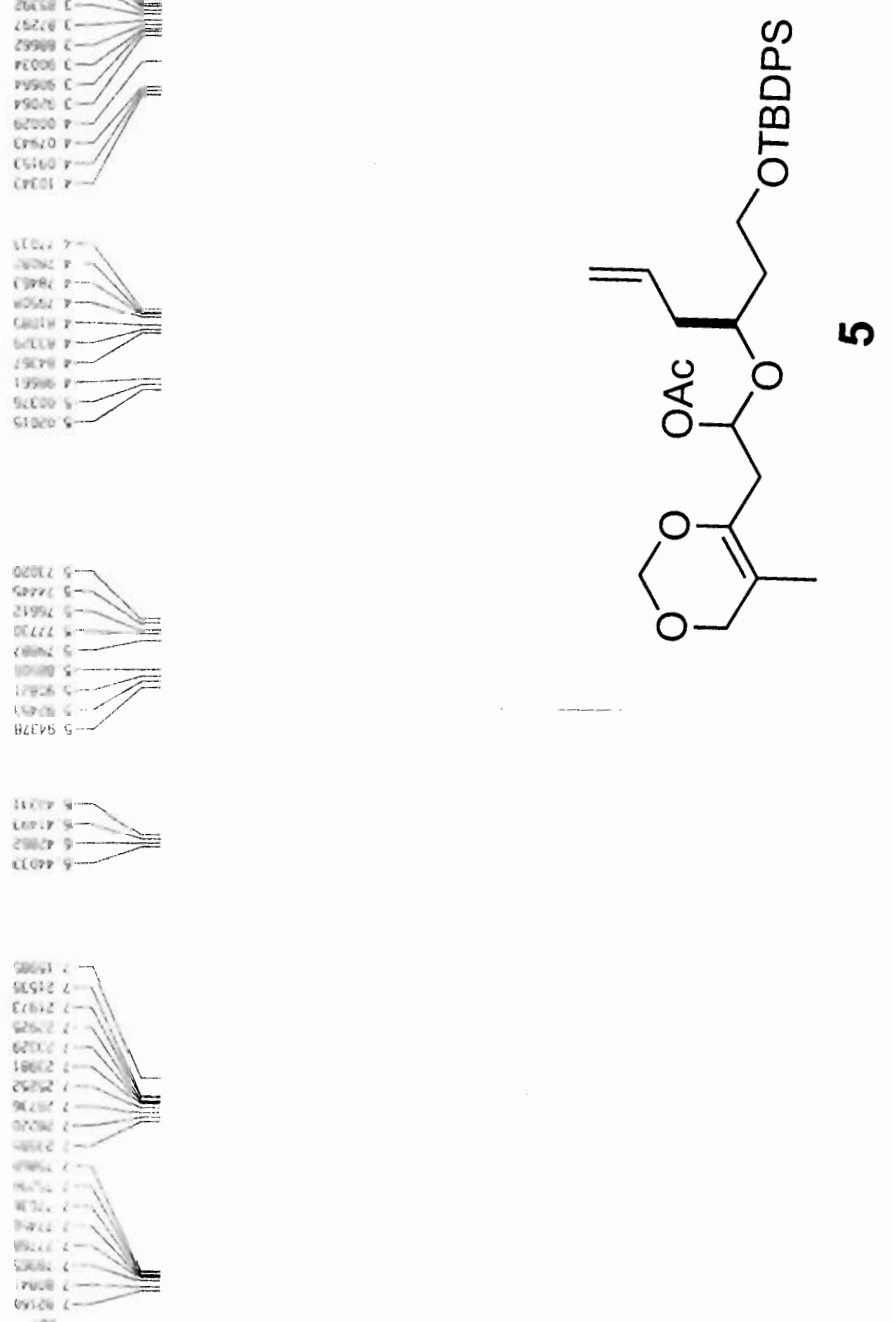

$\infty$
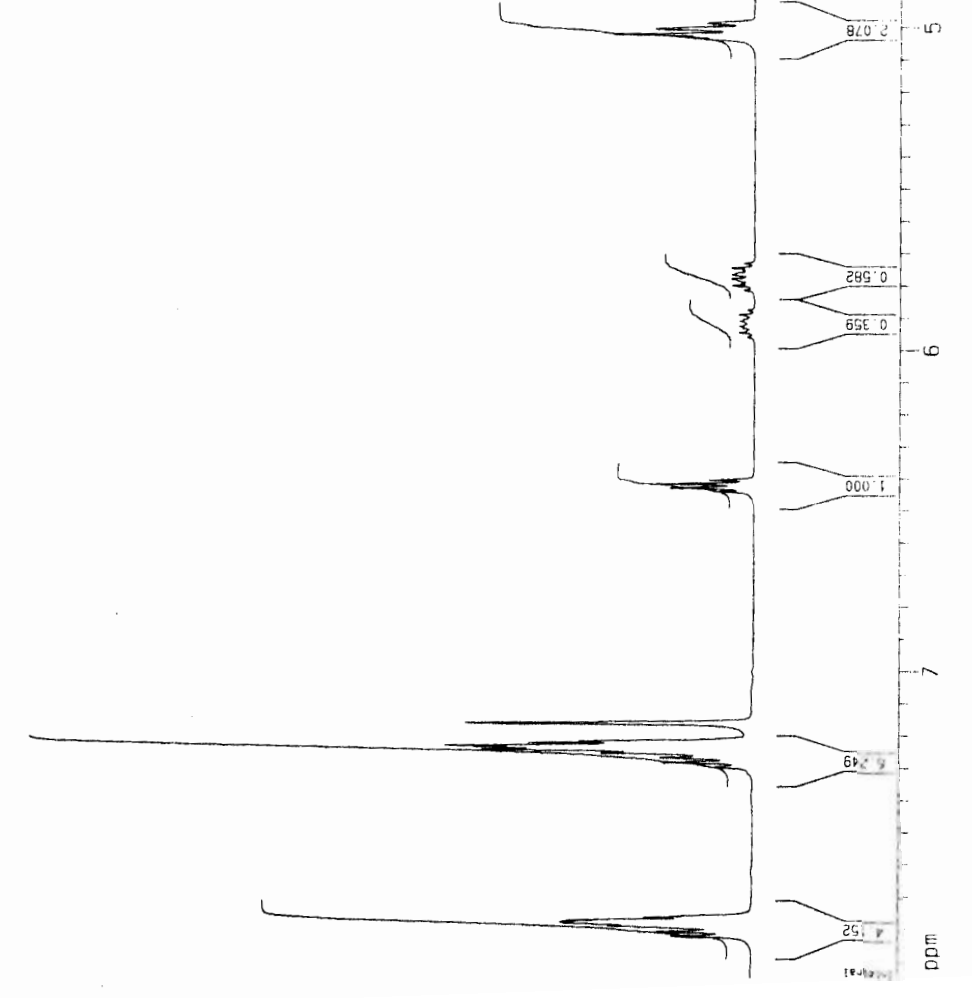


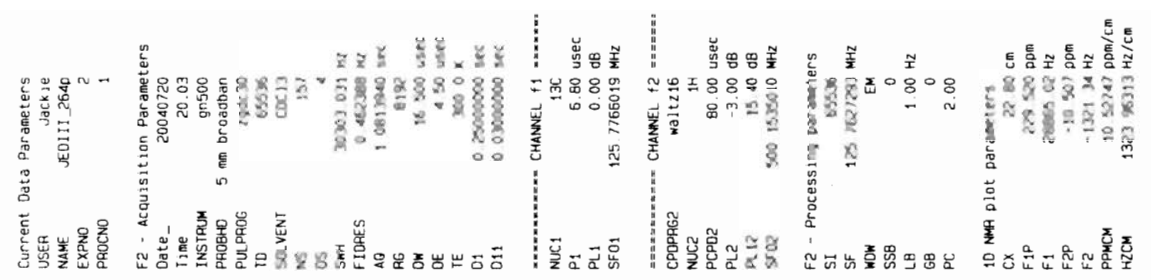

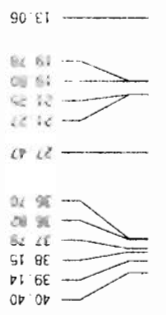

16 og $-1 .-1=$

$7929-1=$

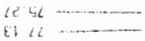

$8606-\ldots$
$00.52-\square$
$0696-\square$
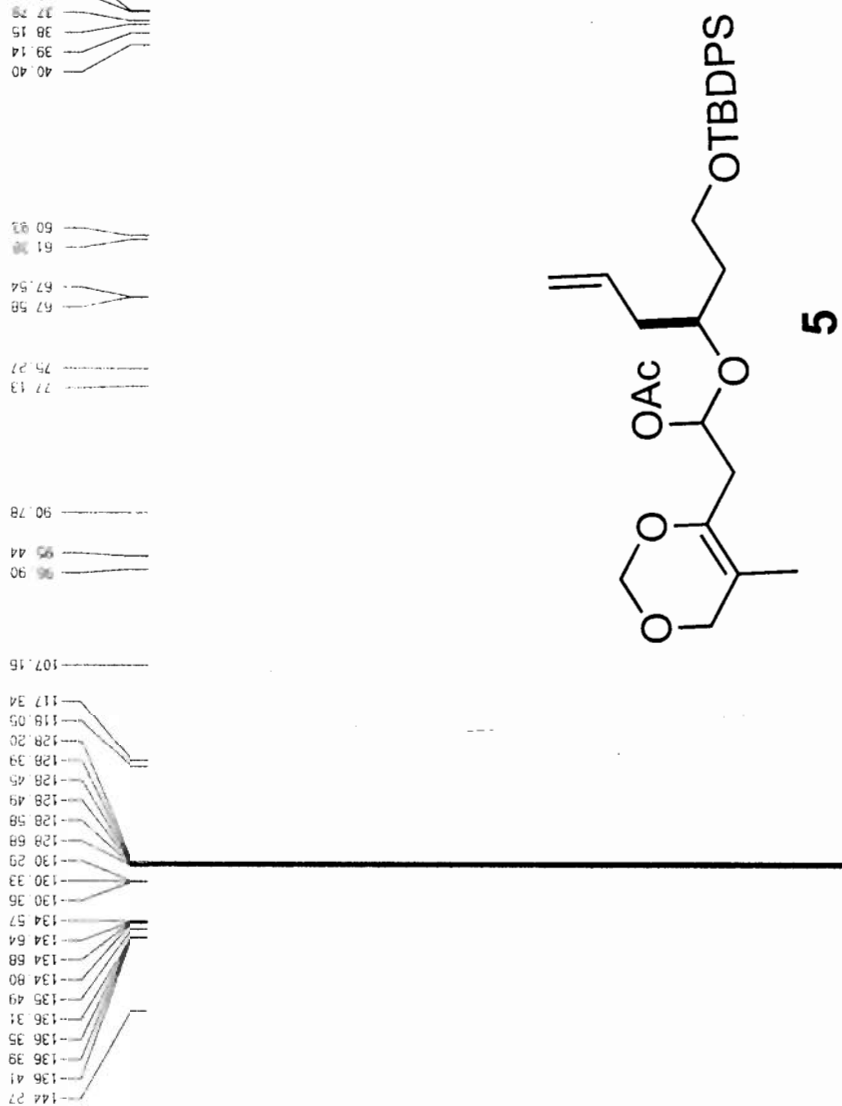

م

0

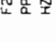



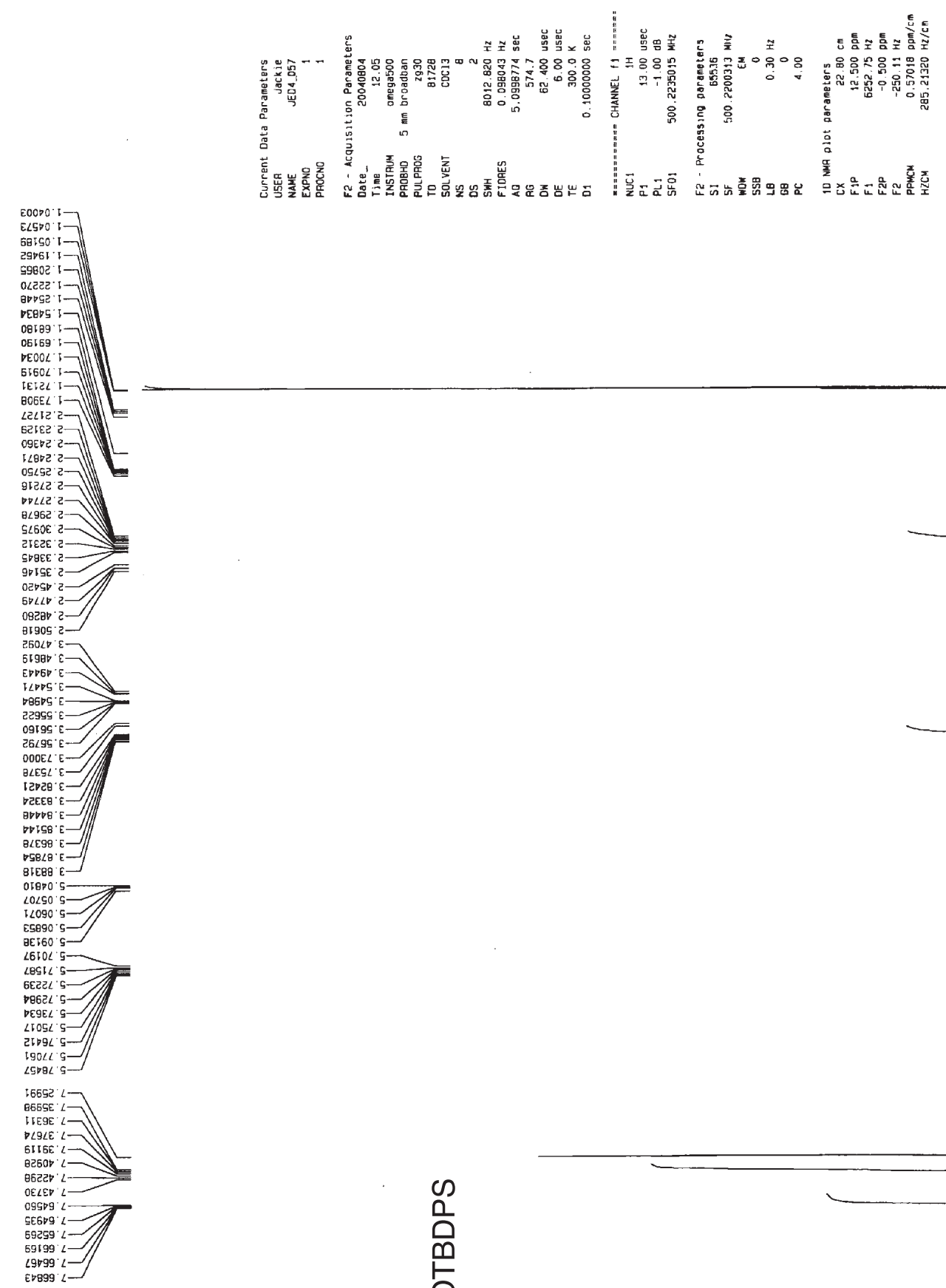

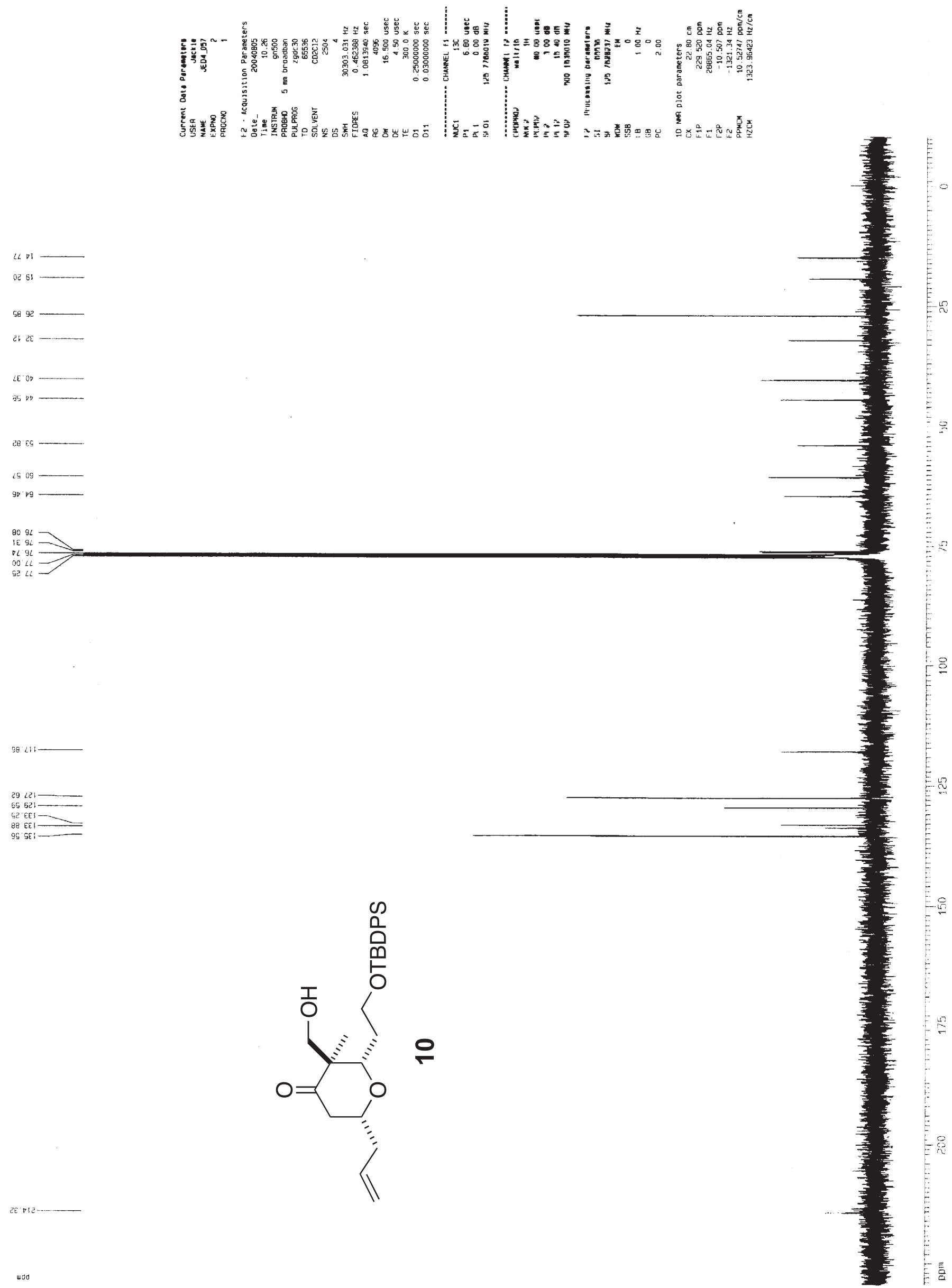


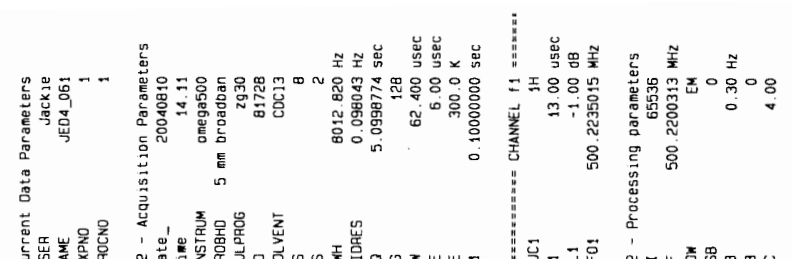

$2 \angle O L C O$
$2 \angle 0 E 6$

$\begin{array}{lll}2 \angle 8650^{\circ} & 0-1\end{array}$

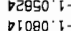

tBiar

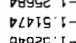

gr925: 1-

टLE\&G :

GSDGS :

29095' 5

EEI $\angle 5^{\circ} \cdot$

$9 \varepsilon 909$ '

94269.

ELPE9 '

प9l9g :-

LEEg9 i-

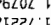

Segel i-

OEDSL $: 1-J$

EREO

68860.2

दारण'ट-

हignis-

1 $\angle 625 \cdot z-$

$\angle S E D T \cdot 2-$

$25625.5-14$

бㄷㄹ. ट-

LEgra' $2-$

56092 z-

$920<2$
SBgs

$\angle \varepsilon \subseteq \angle \varsigma^{\circ} \varepsilon$

$55865^{\circ} \varepsilon-$

$\angle 9 E O L^{\circ} \varepsilon-$

$\angle L 29 L \cdot \varepsilon]$

$0998 \angle \cdot \varepsilon$

$09 \angle 6 i \cdot \varepsilon$

$\varepsilon \varepsilon 900^{\circ} \varepsilon$
в9018' $\varepsilon$

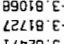

ᄂ $\angle \nabla 2 \theta \cdot \varepsilon$

G58E⿰ $\varepsilon$

$\angle 6858^{\prime} \varepsilon$

(9898

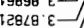

DEDOE. E-

I $65 \angle 1 ;-$

g926) $b-$

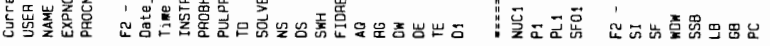

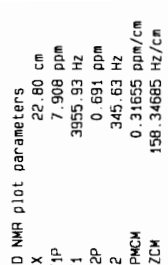

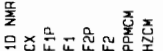

\section{.}

B59 $\angle 6966$

IEI 0 ' $\mathrm{s}-$

$99550 \mathrm{~s}$

2TSEL

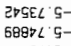

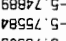

$50 E 9 \angle 2$

$669<5$

or $06<$ L 5

$\checkmark E \angle E L C$

BGEO

ELC10.
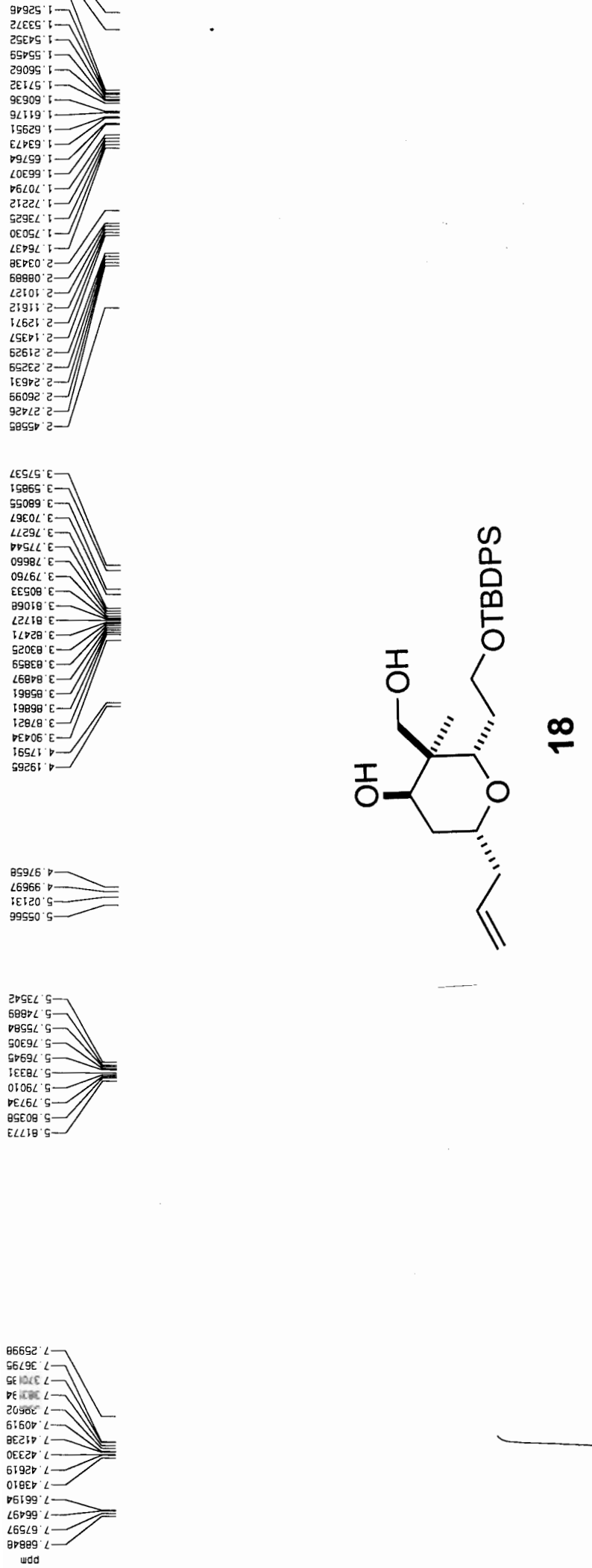

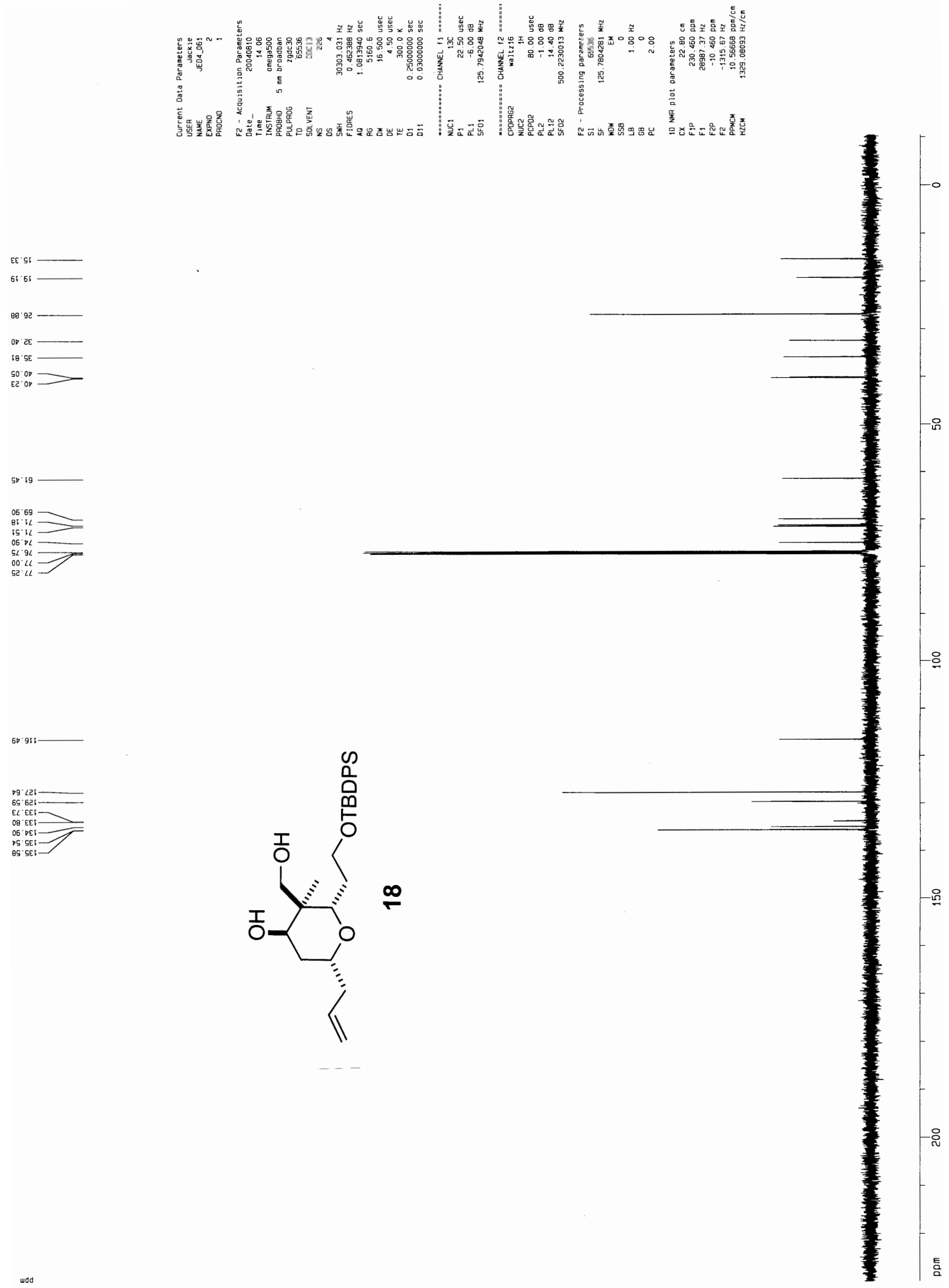


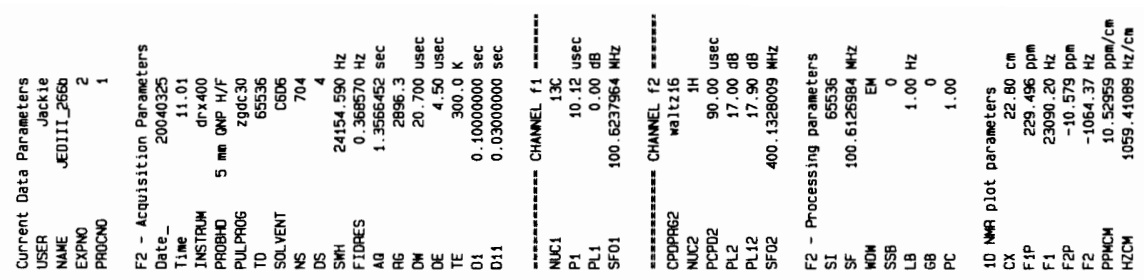

$0.51 \ldots$

6.31

$99^{\circ} \angle 2$

एँ

l's

听化

68. 19

${ }^{80} \mathrm{CL}-$

ar $24=$

6t 08

$99^{\prime} 85$

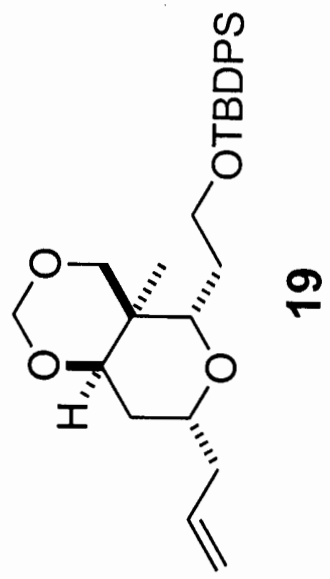

$6911=$

51 825

(c) $82:-$

E9. $821-$

96.961 